\title{
The Effect of Inflow Conditions on the Transition to Turbulence in Large Eddy Simulations of Spatially Developing Mixing Layers
}

\author{
W. A. McMullan*, S. Gao, C. M. Coats \\ Dept. of Engineering, University of Leicester, University Road, Leicester, England, \\ LE1 $7 R H$
}

\begin{abstract}
Large Eddy Simulations (LES) of spatially developing turbulent mixing layers have been performed for flows of uniform density and Reynolds numbers of up to 50,000 based on the visual thickness of the layer and the velocity difference across it. On a fine LES grid, a validation simulation performed with a hyperbolic tangent inflow profile produces flow statistics that compare extremely well with reference Direct Numerical Simulation (DNS) data. An inflow profile derived from laminar Blasius profiles produces a flow that is significantly different to the reference DNS, particularly with respect to the initial development of the flow. When compared with experimental data, however, it is the boundary layer-type inflow simulation produces the better prediction of the flow statistics, including the mean transition location. It is found that the boundary layer inflow condition is more unstable than the hyperbolic tangent inlet profile. A suitably designed coarse LES grid produces good predictions of the mean transition location with boundary layer inflow conditions at a low computational cost. The results suggest that hyperbolic tangent functions may produce unreliable DNS data when used as the initial condition for studies of the transition in the mixing layer flow.
\end{abstract}

Key words: Large Eddy Simulation, Mixing Layer, Transition, Inflow Conditions $P A C S: 76 \mathrm{~F} 06,76 \mathrm{~F} 10,76 \mathrm{~F} 65$

\footnotetext{
* Corresponding author. Current address: Dept. of Aeronautical and Automotive Engineering, Loughborough University, Loughborough, LE11 3TU. Fax: +44 (0)1509 227275

Email address: wam3@le.ac.uk (W. A. McMullan).
} 


\section{Introduction}

The mixing layer flow and its transition to turbulence are much-studied areas of fluid dynamics, as the flow configuration is considered to be of fundamental importance in practical engineering applications. Whilst the fully-developed layer was initially considered to be an essentially random turbulent flow-field, the discovery of coherent motions embedded within the flow [1], [2], prompted significant interest in the dynamics of these structures [3], [4], [5], [6], [7]. It was noted that, at moderate Reynolds number at least, the pairing of KelvinHelmholtz $(\mathrm{K}-\mathrm{H})$ vortices caused the mixing layer to grow [2], and at a sufficiently high Reynolds number (based on the visual thickness of the layer and the velocity difference across it) such a pairing interaction would cause the layer to undergo a transition into a turbulent state [8]. The vortex amalgamation events and transition to turbulence were found to be a function of the momentum thickness of the high-speed side boundary layer, $\theta_{1}$. Detailed investigation of the transition process in the mixing layer flow has shown that the mixing transition occurs as a result of vortex amalgamation, once the local Reynolds number is high enough to support an extended energy cascade [8]. It was also noted that because the transition is triggered by an amalgamation event, it does not take place in a fixed position, but rather moves on a cyclic basis dependent upon the location of the triggering interaction. This process is generally complete by a Reynolds number of $10^{4}[9]$. Along with the amalgamation process, the presence of the secondary, streamwise-orientated structures is required to produce the transition to turbulence. These structures manifest themselves as streaks in plan-view shadowgraph images of the mixing layer [4], and give rise to the 'mushroom-shaped' eruptions on the cores of the primary vortices and their interconnecting braids [3]. Forcing of low-frequency $\mathrm{K}-\mathrm{H}$ modes tends to prevent the formation of these vortices and delays the onset of turbulence within the mixing layer [10]. Coupled with the presence of the coherent structures in the turbulent flow, the transition to small scales in the mixing layer is also accompanied by a step increase in the amount of molecularly-mixed fluid present in the layer [4], [11]. As a result, the transition in mixing layers is more commonly referred to as the mixing transition.

Some of the first studies into the mixing layer have shown that the flow displays a hypersensitivity to its initial conditions, as similar experiments performed in different apparatus have produced growth rates of the single-stream flow that vary by up to $30 \%$ [12], [13]. These discrepancies can at least partly be attributed to the state of the separating boundary layer at the splitter plate edge [14].

Numerical simulation provides a controlled environment in which to test the sensitivity of the flow to its initial conditions, and to recreate the transition to turbulence. The high computational cost involved in simulating the spa- 
tially developing flow has led to many instances of temporally-evolving mixing layer simulations, where periodic boundary conditions are imposed in both the stream- and span-wise flow directions. These simulations have met with some success, with the roll-up of the fundamental instability [15], the pairing of primary vortices [16] and the onset of the transition to turbulence captured [17] all captured by DNS.

Recent advances in computing power have permitted the simulation of spatiallydeveloping mixing layers that are of practical relevance. Many such simulations are performed in two-dimensions [19], [20], [21], [22], [23], confining the flow to development from $\mathrm{K}-\mathrm{H}$ instability modes, as the lack of a spanwise dimension prevents the formation of secondary streamwise vortices that are necessary for the transition process to occur [24]. This artificial confinement results in predicted entrainment ratios that are markedly different to those found in the real flow [25]. A recent study by Wang et al. [26] has shown that three-dimensional DNS of the spatially-developing flow is now possible, offering a highly detailed view of the transition process. Studies of the effect of subgrid-scale modelling in Large Eddy Simulations (LES) have been performed for both temporally- and spatially-developing simulations [27], [28], with the spatial flow being much less sensitive to the subgrid-scale model on a reasonably fine grid. The vast majority of spatially-developing simulations have been confined to the pre-transition region of the flow [28], [29], with no data available on the performance of LES in predicting the fully turbulent flow.

Given the success of numerical simulations in predicting many of the features of the mixing layer, it is surprising that no published studies have simulated the transition process of the spatially-evolving layer for direct comparison with either experimental or DNS data. The high computational cost involved in the simulations performed by Wang et al. [26] requires that more coarse grids are used in a parametric study of the transition to turbulence in mixing layers. In this research, a series of Large Eddy Simulations will be performed to assess the capability of the method to capture the transition to turbulence in the mixing layer. The research code will be validated against existing DNS data presented in [26] on relatively high-resolution grids. In addition, two typically-used inlet conditions will be tested and their ability to predict the bulk properties and the mean transition length of reference experimental data will be assessed. The effect of grid resolution on the flow will also be analysed.

\section{Numerical Methods}

The research code has previously been used to simulate transition in jet flows [30] and turbulent near-wake flows [31]. The code is based on the spatially fil- 
tered analytic equations for conservation of momentum and mass of a uniform density fluid, given by

$$
\begin{aligned}
& \frac{\partial \bar{u}_{i}}{\partial t}=-\frac{\partial \bar{p}}{\partial x_{i}}+\frac{\partial}{\partial x_{j}}\left(-\bar{u}_{i} \bar{u}_{j}+2 \nu \bar{S}_{i j}\right) \\
& \bar{S}_{i j}=\frac{1}{2}\left(\frac{\partial \bar{u}_{i}}{\partial x_{j}}+\frac{\partial \bar{u}_{j}}{\partial x_{i}}\right) \\
& \frac{\partial \bar{u}_{i}}{\partial x_{i}}=0
\end{aligned}
$$

These equations are discretised on a staggered mesh as per Roache [32]. The viscosity $\nu$ can consist of both a molecular and a subgrid component, $\nu=$ $\nu_{m}+\nu_{s g}$, if a subgrid-scale model is used. The governing equations are advanced in time using a second-order Adams-Bashforth technique. The pressure field is solved implicitly through the use of the continuity equation by using a novel multi-grid method to maximise the speed of convergence.

The dynamic procedure of Germano and Lilly [33], [34] is employed to model the unresolved scales of motion, given by

$$
C=\frac{1}{2} \frac{\left\langle L_{k l} M_{k l}\right\rangle}{\left\langle M_{i j} M_{i j}\right\rangle}
$$

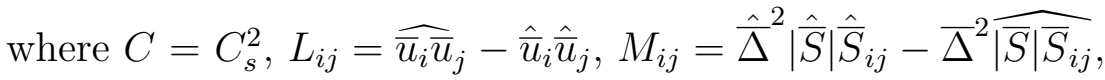

and the angled brackets denote an averaging procedure in a homogeneous direction. The test filter width is given the value $\hat{\bar{\Delta}}=2 \bar{\Delta}$, where $\bar{\Delta}$ is the characteristic filter-width of the computational cell, nominally obtained from the cube-root of the cell volume. In the present study the averaging is performed in the spanwise direction. The model allows for negative values of $C$, known as backscatter. These negative values can be large and persist for long periods of time causing numerical instability, and as such the total viscosity is set to zero when negative values are computed. In regions where positive values of $C$ are reported, the subgrid-scale viscosity is obtained using

$$
\nu_{s g}=C \bar{\Delta}^{2}|S|
$$

following Smagorinsky [35], with $|S|=\left(2 S_{i j} S_{i j}\right)^{1 / 2}$. 
A passive scalar is also introduced into the flow domain, and its motion is governed by the equation

$$
\frac{\partial \bar{T}}{\partial t}=\frac{\partial}{\partial x_{i}}\left(-\bar{u}_{i} \bar{T}+\alpha \frac{\partial \bar{T}}{\partial x_{i}}\right)
$$

where $\alpha$ is the diffusivity, which can contain both a molecular and a subgrid component, $\alpha=\alpha_{m}+\alpha_{s g}$, if a subgrid-scale model is used. The Schmidt number of the flow, $S c=\nu / \alpha=0.7$ in all cases. The scalar is discretised at the cell centre, and a second order upwinding scheme is used to calculate the scalar flux between cell faces, following the work of Gao and Voke [36] reducing overshoots in the scalar field to less than 5\%. Time advancement is again achieved through the Adams-Bashforth technique.

The outflow condition is of an advective form, given by the equation

$$
\frac{\partial \bar{u}_{x}}{\partial t}=-U_{c} \frac{\partial \bar{u}_{x}}{\partial x}
$$

where the derivative $\partial \bar{u}_{x} / \partial x$ is necessarily one-sided, and $U_{c}$ is the mean streamwise velocity at the outflow plane. A previous study using the code has shown that this condition is entirely passive [37]. The outflow condition for the passive scalar is treated in a very similar manner.

\section{Reference Simulation}

A reference LES is performed against the DNS calculations of Wang et al. [26]. The High-Resolution (HR) grid used in this study contains an order of magnitude fewer cells than that of Wang et al. and is not intended to provide a direct DNS comparison of that data. The emphasis of the HR simulation is to produce an LES solution that predicts the trends of the DNS data, and to provide a reference for the varying inlet condition that will be studied in Section 4.

The inflow condition for the mixing layer is specified as an analytical hyperbolic tangent (HT) function given by

$$
\bar{u}(y)=\frac{U_{1}+U_{2}}{2}+\frac{U_{1}-U_{2}}{2} \tanh \left(\frac{2 y}{\delta_{i}}\right)
$$

where $U_{1}$ and $U_{2}$ are the high- and low-speed side freestream velocities respectively, and $\delta_{i}$ is the initial vorticity thickness of the layer which can be 
calculated using the expression

$$
\delta_{\omega}=\frac{U_{1}-U_{2}}{(\partial \bar{u} / \partial y)_{\max }} .
$$

Velocity profiles for the cross-stream and spanwise components are set to zero at the inflow plane. White-noise perturbations generated from a pseudorandom number generator are superposed onto the inflow velocity profiles, with a peak value of $0.5 \%$ of the freestream velocity. The fluctuations at the inflow plane are superposed at every time step, and have a lengthscale that is of the order of the local grid-filter length, $\bar{\Delta}$. Table 1 details the dimensions of the HR grid along with that used in the reference simulation [26]. The grid-spacing is uniform in the streamwise and spanwise directions, with the cross-stream grid spacing stretched very weakly in the region away from where large fluctuations in the flow-field are expected to occur. The grid is non-dimensionalised with respect to the wavelength of the most unstable mode of the inlet profile given by $\Lambda=14.132 \delta_{i}$, obtained from linear stability theory.

The inflow Reynolds number, based on the velocity difference, $\Delta U$, and the initial vorticity thickness, $\delta_{i}$, is 700 , with the velocity ratio, $R$, of the mixing layer defined as

$$
R=\frac{U_{1}-U_{2}}{U_{1}+U_{2}}
$$

taking a value of 0.33 . The time step for the simulation is set to $7 \times 10^{-7} \mathrm{~s}$ and is run for $60,000 \Delta \mathrm{t}$. The simulation requires approximately 520 hours to complete on a single $2.2 \mathrm{GHz}$ AMD Opteron processor. Statistics and flow-field outputs are obtained over the final $60 \%$ of the run. The simulation is denoted WHTHR.

Representative spanwise-averaged contour plots of the passive scalar and spanwiseaveraged vorticity are given in Figure 1 . The flow is dominated by coherent vortices whose number density reduces with downstream distance, implying that interactions occur between them. Small scales of motion appear beyond $x=14 \Lambda$, which suggests that the flow undergoes a transition to turbulence within the computational domain. These images bear close resemblance to those presented in the reference simulation [26], as K-H type vortices emerge from the vortex sheet at $5 \Lambda \lesssim x \lesssim 8 \Lambda$, a pairing of vortices occurring at $8 \Lambda \lesssim x \lesssim 16 \Lambda$, and a further generation of interactions occurring between $16 \Lambda$ and the downstream end of the computational domain. An iso-surface of total vorticity in the flow is shown in Figure 2, and the secondary streamwise vortices that exist in the braid region between the primary spanwise rollers can clearly be seen. Small scales can also be seen to develop in the flow. 
The momentum thickness of the flow, calculated using the definition

$$
\theta=\int_{-\infty}^{\infty}\left(U_{1}-\bar{u}(y)\right)\left(\bar{u}(y)-U_{2}\right) d y
$$

is shown in Figure 3. Excellent agreement with the reference simulation is obtained. The evolution of the frequency spectra with streamwise distance shown in Figure 4 also compares favourably with the reference DNS, as the $-5 / 3$ roll-off in the exponent, typical of the fully-developed flow, is reached at $x=10 \Lambda$. The low number of samples taken to produce the frequency spectra results in the somewhat non-converged nature of the plots shown in all the HR grid spectral plots presented in this article. Downstream of this location all of the spectra have a similar form.

The subgrid-scale model reports a maximum ratio of subgrid to molecular viscosity, $\nu_{s g} / \nu_{m}=5.1$, at a streamwise location of $x=17.2 \Lambda$, well downstream of the transition to turbulence in the flow. A snapshot of this ratio, taken at the mid-span of the domain, is shown in Figure 5 for this case. As the value of this ratio is typically less than 1 in the laminar region of the flow, the Germano-Lilly model does not excessively damp out instabilities in the flow and highlights the potential usefulness of LES in simulating the turbulent mixing layer originating from initially laminar conditions. Analysis of such instantaneous subgrid-scale to molecular viscosity ratio plots show that pairing interactions cause a local increase in its value as the model attempts to dissipate the small-scales produced in the interaction. In the laminar region these values quickly drop to less than 1 once the interaction has completed. If the pairing interaction triggers the transition to turbulence, the value of the ratio rises to $\sim 3-5$ and remains in this region for the remainder of the computational domain. The above results suggest that the current grid is capable of capturing the large-scale evolution of the flow extremely well, with the removal of the fine scales of motion from the flow not significantly altering the overall flow dynamics.

A measure of the growth of the mixing layer can be provided by calculating the vorticity rate of the flow defined as

$$
\delta_{\omega}^{\prime}=\frac{d\left(\delta_{\omega}\right)}{d x}=\frac{d\left(\delta_{\omega}\right)}{x-x_{0}}
$$

where $x_{0}$ is the virtual origin of the mixing layer. For the current simulation, the vorticity rate $\delta_{\omega}^{\prime}=0.049$, a value that is at the lower limit of the correlation supplied by Brown and Roshko [1]. 


\section{Boundary Layer Inflow Simulation}

The hyperbolic tangent function defined by equation 8 is frequently used as the initial condition in both temporally- and spatially-evolving mixing layer simulations [19], [27], [29]. In the real flow, however, there exists a small wakedeficit caused by the influence of the splitter plate on the flow - a feature that is not modelled in equation 8. Previous studies have shown that this wake-like region can have a significant effect on the evolution of the flow [25], [38], [40], hence the simulation above is now repeated using an inflow condition that accounts for the wake-deficit. Accurate prediction of the transition location in turbulent shear flows is necessary in many practical engineering flow configurations, hence the effect of any modelling assumptions on this parameter should be highlighted.

The boundary layer inlet condition is obtained from the Blasius solution to the laminar boundary layer equations. The momentum thickness of the highspeed side boundary layer is set to $\theta_{1}=\delta_{i} / 4$, following Monkewitz and Huerre [39], as it is known that the vorticity from the high-speed stream is most receptive to high-frequency disturbances and hence determines the frequency of the fundamental K-H mode [24]. The momentum thickness of the low-speed side boundary layer is set to $\theta_{2}=2 \delta_{i} / 5$. A comparison of the inflow conditions used in the present and validation simulations above is shown in Figure 6. All other simulation parameters are held constant with respect to case WHTHR to ensure a direct comparison between the two simulations. The boundary layer inflow condition simulation is denoted WBLHR.

The vorticity rate of the WBLHR, $\delta_{\omega}^{\prime}=0.056$, is almost $15 \%$ higher than that of case WHTHR, and in much better agreement with the correlation of Brown and Roshko [1]. The maximum value of the eddy- to molecular viscosity ratio, $\nu_{s g} / \nu_{m}=5.5$, is slightly higher than case WHTHR and is again found far downstream in the turbulent flow. Viscosity ratio values in the laminar flow regime are also typically less than 1. Spanwise-averaged passive scalar and spanwise vorticity plots at an arbitrary instant in time are shown in Figure 7 for case WBLHR. From Figures 1(b) and 7(b), it is evident that the change in the inlet condition has a significant effect on the initial development of the flow. The emergence of vortices from the vortex sheet occurs much further upstream in this simulation, in the region of $2 \Lambda \lesssim x \lesssim 4 \Lambda$, with subsequent generations of interactions occurring between $6 \Lambda \lesssim x \lesssim 10 \Lambda$ and $14 \Lambda \lesssim x \lesssim 20 \Lambda$ respectively. Inspection of the flow-field shows that fine-scale motions appear within the flow at $x \approx 9 \Lambda$, indicating that the flow undergoes a transition to a turbulent state. An iso-surface of the total vorticity in the flow is shown in Figure 8 - the primary rollers develop further upstream in the simulation than in the HT inlet condition simulation, and the secondary vortices are again clearly visible in the flow. 
The frequency spectra obtained from the simulation along the centreline of the mixing layer are shown in in Figure 9. The differences in the evolution of the frequency spectra between cases WBLHR and WHTHR are consistent with what is noted in the instantaneous flow-field images. At $x=2 \Lambda$ the spectrum has a steep exponent with two well-defined peaks, showing the action of subharmonics of the fundamental K-H instability. The spectra also show that the flow approaches a fully turbulent state as far upstream as $x=6 \Lambda$, with all the spectra downstream of this location displaying the $-5 / 3$ exponent in the rolloff. As no comparative experimental data are available for the flow conditions simulated in the previous sections, it is not possible to determine which inlet condition produces the most accurate prediction of the flow and the average location at which it undergoes transition to turbulence. Further simulations will now be performed at conditions that match available experimental data [41].

\section{Comparisons With Experimental Data}

A previous, unpublished experimental study into coherent structure evolution in plane mixing layers provides the reference data for the current simulations [41]. As part of the experiments, a parametric study of the transition to turbulence in mixing layers was performed, with the transition location of the flow recorded to a high degree of accuracy over a large range of both the velocity ratio parameter, $R$, and Reynolds number. Two distinct flow types were studied; in the first, air was used in both freestreams ('HW') to permit the use of hot-wire anemometry to obtain velocity distribution measurements and frequency spectra. In the second, a mixture of helium and argon was used as the gas in the high-speed stream, and air in the low-speed stream ('F' and 'FP'), which had the effect of producing a gas of uniform density within the mixing layer. Such experiments were performed in order to allow side- and plan-view schlieren and shadowgraph images to be obtained for flow visualisation. Cinefilms of these visualisations were made to study the evolution and interaction of coherent structures within the flow on a time-resolved basis. Digital Image Processing was performed on the sequences to improve coherent structure eduction, and is was found that the abrupt disappearance of the streamwise streaks and appearance of small-scale eddies in the plan-view shadowgraph images coincided with the appearance of small scales in shadowgraph side-view images. The appearance of the small scales always coincided with a triggering interaction between laminar vortices. These images demonstrated that the laminar and turbulent regimes of the flow were unambiguous and the transition in the flow could easily be noted in them. In agreement with previous experimental data, these experiments demonstrated that the instantaneous transition location moved up- and down-stream according to the position of 
the triggering interaction between vortices [8] and as such the values obtained from the experiments should be considered as a temporal mean. Additionally, the mean streamwise velocity fluctuation $u^{\prime}$ was recorded along the centreline of the layer, with the quantity approaching a given value once the flow had become turbulent [42].

\subsection{Simulation Setup}

In Table 2, the flow conditions of a small cross-section of the experiments performed by D'Ovidio [41] are outlined. The transition location is non-dimensionalised by the so-called pairing parameter defined by Huang and Ho [8]. The pairing parameter is given by

$$
x_{i}^{*} \approx \frac{R x}{30 \theta_{1}}
$$

with the first, second and third amalgamation events occurring at $x_{i}^{*}=4,8$, and 16 respectively. In the reference experiment the transition to turbulence normally occurred with the second generation of vortex interactions, such that $x_{i}^{*} \approx 8$ for all cases, confirming the data of Huang and Ho [8], and Jimenez [42] for comparable Reynolds numbers.

Simulations are performed for these flow conditions using both the hyperbolic tangent and boundary-layer type inlet conditions in order to assess the impact of the varying inflow condition on the mean transition location. Mean flow statistics were recorded for experiment HW9 and a simulation of this case is performed to compare these quantities. The lower freestream velocities of these flows permit the simulation time-step to be increased to $1 \mu \mathrm{s}$, without the maximum reported Courant number exceeding 0.3. All other simulation parameters match those of WHTHR and WHTBL. The largest Reynolds number recorded in the simulations is $R e \approx 50,000$ in case HW9, based on the velocity difference across the layer and its visual thickness.

\subsection{Simulation Results}

Flow statistics obtained from the simulation are transformed into a nondimensional co-ordinate system, $\eta$, defined by

$$
\eta=\frac{y-y_{0.5}}{x-x_{0}}
$$


where $y_{0.5}$ is the lateral position at which the streamwise velocity is equal to the average of the freestream values.

The mean and root mean squared (r.m.s.) flow statistics from simulations of HW9 are shown in Figure 10. The mean streamwise velocity fits well with the experimental data, whilst the r.m.s. streamwise velocity fluctuation of the HT inflow simulation has a slightly narrower distribution than the experimental data, implying that the spreading rate of this simulation is lower than the real flow. The BL inflow simulation statistics, however, compare more favourably with the experiment. No cross-stream or spanwise velocity fluctuation r.m.s. data are available from the experiment, but the simulation data again shows a slower spreading of the mixing layer in the HT inflow condition simulation.

The spreading of the mixing layer simulations is quantified in Figure 11. The HT inlet condition simulations have a vorticity rate that is significantly lower than the experiment, whilst the BL inlet condition produces mixing layers that spread at a rate much more consistent with the real flow. As all simulation parameters aside from the inlet condition were held fixed, the observed discrepancy in the growth rate of the simulated mixing layers is a direct consequence of the applied inlet condition. Representative spanwise-averaged spanwise vorticity plots of case HW9 from both inlet condition simulations are shown in Figure 12. As has been noted in the validation simulations, an extended vortex sheet is present in the HT inlet simulations, from which primary vortices emerge and proceed downstream. In the BL inlet simulation this vortex sheet is much shorter, with the emergence of vortices occurring much further upstream.

The transition length of the simulated flows is determined using numerical analogies of the three experimental techniques. The first, and most accurate method is through the analysis of sequences of instantaneous, spanwiseaveraged images such as those shown in Figures 1, 7, and 12. In each simulation, a series of 200 images is stored to disk, and the position at which the amalgamation that precipitates the transition to turbulence is noted. An average of the positions is then taken to calculate the mean transition location. In Figure 12 the transition in the flow has occurred at $x=0.08 \mathrm{~m}$ and $x=0.055 \mathrm{~m}$ in the HTHR and BLHR simulations respectively, as the distribution of vorticity in the flow has abruptly changed from being concentrated within the laminar vortex cores into a more uniform distribution within the post-transition coherent structures downstream of these positions. The appearance of these fine scales coincides with a pairing of primary vortices, an observation consistent with previous experimental data [8]. The instantaneous transition location within the flow moves up- and down-stream by a few centimetres, according to the position of the triggering amalgamation. 
The second method is through the analysis of frequency spectra recorded along the centreline of the layer at several streamwise locations, as shown in Figure 13 for case HW9BLHR. The steep roll-off in the spectra upstream of $x=0.04 \mathrm{~m}$ shows that the flow is laminar in this region. As the flow proceeds downstream, the roll-off exponent converges towards the $-5 / 3$ slope representative of turbulent flow. The evolution of the roll-off is progressive because the transition location moves around with the triggering pairing interaction. As the instantaneous transition location can move up- and down-stream by a few centimetres this statistical approach to the calculation of the mean transition location is less accurate than the analysis of the instantaneous flow-field images. Finally, the evolution of the streamwise velocity fluctuation $u^{\prime}$ for cases HW9 and F9 are shown in Figure 14. The shoulder in the profiles of HW9BLHR and F9BLHR at $x \approx 0.02 \mathrm{~m}$ is caused by the increase in velocity fluctuation due to the roll-up of the layer into primary vortices, with the peak in the profiles corresponding to the mean location of the first vortex amalgamation. The counterpart HT inlet simulations do not display this shoulder, and the convergence of $u^{\prime}$ is delayed by a significant streamwise distance in these cases.

The calculated mean transition lengths for the simulated flows are shown in Table 3, expressed in terms of the pairing parameter, $x_{i}^{*}$. The transition of the mixing layer occurs at a Reynolds number of 10,000-20,000, consistent with past research [8], [9]. In all simulations the transition to turbulence is normally completed by the second generation of pairings between the primary spanwise-orientated vortices. When compared with the transition locations recorded in the experiment (Table 2) it is clear that the BL inlet condition simulations produce data that agrees very well with the experiments, whilst the HT inlet condition simulations produce mean transition lengths that are too far downstream.

\section{Coarse Grid Simulations}

The prohibitively expensive computational cost of the HR simulations will prevent such a fine LES grid being used in studies of laminar-turbulent shear layers that involve sub-grid mixing and chemical reactions. Thus the grid is coarsened to test the effects of mesh resolution on the bulk evolution of the flow. As the boundary layer-type inflow condition produces the most accurate prediction of the flow evolution on the HR grid, only this inlet condition is considered in this set of simulations.

Simulations performed on a grid that can resolve the initial vorticity thickness, $\delta_{i}$, but cannot resolve the initial momentum thickness of the high speed side boundary layer, $\theta_{1}$, have been performed and give poor agreement with the 
experimental data [43]. Given this finding a further mesh is designed that shares the same domain extent and initial grid spacing as the High Resolution grid, but takes advantage of grid stretching to reduce the total number of nodes within the domain. The grid is stretched in the $x$ - and $y$-directions using a geometric expression with the cell-expansion ratio set at 1.01, and the $z$ - grid-spacing remains constant. The mesh parameters are given in Table 1. On this Intermediate Mesh (IM) the simulations are performed for $120,000 \Delta \mathrm{t}$ with a time step of $1 \mu \mathrm{s}$. Flow statistics are gathered over the final $75 \%$ of the runs.

The mean and r.m.s. flow statistics in Figure 15 compare reasonably well with the available experimental data, with the $u^{\prime}$ profiles suggesting that the simulated layer has a slightly higher spreading rate than the experiment. The contours of spanwise-averaged spanwise vorticity in Figure 16(b) show that the coarsening of the grid removes the small-scales of motion embedded within the coherent structures in the turbulent flow, although the roll-up of the flow into K-H vortices remains largely unchanged. Similarly, the scalar field in Figure 16(a) has a smoother appearance within the coherent structures. The visual thickness of the layer on this Intermediate Mesh, however, has a similar magnitude to the layer produced on the HR grid in Figure 1 . The vorticity rate of the flows, given in Figure 17, is slightly over-predicted on the Intermediate Mesh, due to the coarseness of the cross-stream grid away from the plane of the splitter plate.

The mean transition location of the flow is again calculated using the methods described above, with 900 frames of flow outputs available for analysis from the IM simulations. Representative plots of $u^{\prime}$ are shown in Figure 18, with the evolution of the quantity on the Intermediate Mesh agreeing favourably with the BLHR results. The peak fluctuations are lower on the Intermediate Mesh due to the subgrid-scale model removing fluctuations in the flow on the coarser grid. The mean transition locations are given in Table 4 and are very similar to both the BLHR simulations and the experimental data. The Reynolds number at which the transition occurs also falls within the limits defined by experiment.

\section{Further Discussion}

The results presented in the preceding sections have significant implications for DNS benchmarking of the mixing layer and its modelling through LES techniques. It is apparent from the presented results that the hyperbolic tangent inflow condition provides an inadequate description of the initial development of the mixing layer flow, observed in both its delayed instability to disturbance modes and its subsequent delayed transition to turbulence. Stored flow-field 
images such as those in Figures 1 and 7 are analysed in more detail to quantify this discrepancy. The flow near the inlet plane is visualised with streamlines that are produced in a Lagrangian frame of reference, with the streamwise velocity field brought to rest through the sum of $\bar{u}-U_{c}$, where $U_{c}=\left(U_{1}+U_{2}\right) / 2$ is the convection velocity of the structures. An example of the flow-field near the inlet plane visualised in this manner is shown in Figure 19, taken from case WHTHR. The stored sequences of images from all of the simulations outlined above are used to determine the mean location at which the flow rolls up into discrete vortices and undergoes the first and second generation of vortex amalgamations. The data is normalised against the pairing parameter of equation 5.1 and plotted as a function of the velocity ratio parameter, $R$, in Figure 20. The majority of the boundary-layer inflow condition simulations presented in Figure 20(a) show excellent agreement with the theoretical predictions of the events in the mixing layer, with case WBLHR showing some slight under-estimation of the locations of the first and second generations of vortex amalgamations. The values for all simulations are, however, well within limits defined by experimental data [8], [44]. The data obtained for the hyperbolic tangent inlet conditions simulations is shown in Figure 20(b), along with estimates for the locations of the vortex events in the reference DNS. The results show significant disparity with the theoretical predictions, demonstrating that the imposition of a hyperbolic tangent inlet profile will result in a flow that suffers from a delay in its overall evolution, leading to the poor prediction of the transition location outlined in Table 3.

The results presented here are consistent with a previous study that has shown that the growth and mixing rate in the flow are significantly decreased when a hyperbolic tangent-like error function is used to initialise the layer [38]. The imposition of a hyperbolic tangent function yields a pre-defined selection of a single dominant instability mode that is relatively stable to imposed random fluctuations. It has been reported that the stability of the hyperbolic tangent profile can be significantly modified through the direct forcing of the fundamental instability mode and its subharmonics, derived from Linear Stability Theory [28]. The streamwise distance required to achieve similarity in this case was a factor of 1.5 shorter than in a flow that was perturbed by pseudo-random fluctuations similar in form to those used by Wang et al. [26]. However, real mixing layers contain the wake-like region in their initial velocity profile, and the evidence presented here suggests that including this deficit in the applied inflow condition is vital to ensure that the natural development of the flow is physically realistic. The High Resolution simulations performed in this research therefore indicate that future DNS of mixing layers that originate from laminar boundary layers should use an inlet condition that includes the wake-deficit in the applied streamwise velocity profile.

It has previously been demonstrated that the dominant parameter in the initial development of the flow is the high-speed side boundary layer momentum 
thickness, $\theta_{1}$, as simulations that do not adequately resolve this parameter produce highly inaccurate flow fields and a much-delayed transition to turbulence in the flow [43]. The grid spacing of the Intermediate Mesh in the region just downstream of the inflow plane almost matches that found on the High Resolution grid, but is much coarser in the region downstream of the location where the first generation of amalgamations between $\mathrm{K}-\mathrm{H}$ vortices occurs. Viscous damping effects on the instability of mixing layers are insignificant above Reynolds numbers of $\gtrsim 300$ [47], much lower than the flows simulated here therefore the roll-up and subsequent interactions of the $\mathrm{K}-\mathrm{H}$ vortices are essentially inviscid phenomena. This stipulation is confirmed in Figure 20(a) where the mean locations of the first and second vortex amalgamation events are very close to those predicted in the HR simulations, in spite of the lower grid resolution of the IM runs. It is therefore postulated that LES grids designed for practical engineering applications should be able to capture the processes leading up to the transition in the flow in a reasonably accurate manner if the mesh resolution near the inlet boundary is sensibly specified, and the inlet condition is suitably modelled. Similar conclusions to the above have been found for wall-bounded LES, where the scale of the instability mechanisms are much smaller than those in free-shear flows [48].

\section{Concluding Remarks}

Large Eddy Simulations of spatially developing mixing layers of Reynolds numbers up to 50,000, based on the velocity difference across the layer and its visual thickness, have been performed. Validation simulations performed against a reference DNS [26] show that the bulk features of the flow can be reproduced using a fine-grid LES that contains an order of magnitude fewer cells than the reference DNS. The effect of the inflow condition on the evolution of the flow is tested, with a hyperbolic tangent inflow condition producing a markedly different flow-field to a mixing layer initialised from laminar boundary layer profiles given identical flow conditions.

Further simulations performed demonstrate that, in mixing layers initialised from a hyperbolic tangent profile, the mean location of the transition to turbulence occurs at a point significantly further downstream than found in corresponding experiments [41]. The use of boundary-layer profiles as the inflow condition to the simulation produces mean transition locations that agree very well with experimental data across the range of velocity ratio parameter $R$. The roll-up of the flow into discrete vortices in the hyperbolic-tangent inflow simulations occurs at locations that do not agree well with established theoretical predictions.

Large Eddy Simulations of mixing layers performed on coarse grids have shown 
that adequate resolution of the high-speed side separating boundary layer, $\theta_{1}$, is required to produce simulated flow-fields whose statistical properties agree well with experimental data. Resolving only the initial vorticity thickness, $\delta_{i}$, does not give a simulated flow-field that compares well with experiment. In conjunction with other experimental and simulation data it is postulated that hyperbolic tangent inflow conditions may produce misleading predictions of the location at which transition occurs in the flow in both spatially-developing DNS and LES of mixing layers.

\section{References}

[1] Brown GL, Roshko A. On density effects and large structure in turbulent mixing layers. J. Fluid Mech. 1974; 64:755-816.

[2] Winant CD, Browand FK. Vortex Pairing: The mechanism of turbulent mixing layer growth at moderate Reynolds numbers. J. Fluid. Mech 1974; 63:237-255.

[3] Bernal LP, Roshko A. Streamwise vortex structures in plane mixing layers. $J$. Fluid Mech. 1986; 170:499-525.

[4] Konrad JH. An experimental investigation of mixing in two-dimensional shear flows with applications to diffusion limited chemical reactions. $\mathrm{PhD}$ thesis, California Institue of Technology, 1976.

[5] Koochesfahani MM, Dimotakis PE. Mixing and chemical reactions in a turbulent liquid mixing layer. J. Fluid Mech. 1986; 170:83-112.

[6] Jimenez J, Cogollos M, Bernal LP 1985 A perspective view of the plane mixing layer. J. Fluid Mech. 1985; 152:125-143.

[7] Dimotakis PE, Brown GL The mixing layer at high Reynolds number: large scale structure and entrainment. J. Fluid Mech. 1976; 78:535-560.

[8] Huang L-S, Ho C-M. Small scale transition in a plane mixing layer. J. Fluid Mech. 1990; 210:475-500.

[9] Dimotakis PE. The mixing transition in turbulent flows. J. Fluid Mech. 2000; 409:69-98.

[10] Oster D, Wygnanski I. The forced mixing layer between parallels streams. $J$. Fluid Mech. 1982; 123:91-130.

[11] Breidenthal RE. Chemically reacting turbulent shear layer. AIAA J. 1979; 17:310-311.

[12] Liepmann HW, Laufer J Investigation of free turbulence mixing. NACA tech. note No. 1271947.

[13] Wygnanski I, Fielder HE. The two-dimensional mixing region. J. Fluid Mech. 1970; 41:317-361. 
[14] Batt RG. Some measurements on the effect of tripping the two-dimensional shear layer. AIAA J. 1975; 13:245-247.

[15] Rogers MM, Moser RD. The three-dimensional evolution of a plane mixing layer: the Kelvin-Helmholtz rollup. J. Fluid Mech. 1992; 243:183-226.

[16] Moser RD, Rogers MM. The three-dimensional evolution of a plane mixing layer: pairing and transition to turbulence. J. Fluid Mech. 1993; 247:275-320.

[17] Moser RD, Rogers MM. Mixing transition and the cascade to small scales in a plane mixing layer. Phys. Fluids A 1991; 3:1128-1134.

[18] Balaras E, Piomelli U, Wallace JM. Self-similar states in turbulent mixing layers. J. Fluid Mech. 2001; 446:1-24.

[19] Yang WB, Zhang, HQ, Chan CK, Lin WY. Large eddy simulation of mixing layer. J. Comp. Applied Math. 2004; 163:311-318

[20] Ghoniem AF, Ng K. Numerical study of the dynamics of a forced shear layer. Phys. Fluids 1987; 30:706-721.

[21] Yang WB, Zhang HQ, Chan CK, Lau KS, Lin WY. Investigation of plane mixing layer using large eddy simulation. Comp. Mech. 2004; 34:423-429.

[22] Zhou XY, Pereira JCF. Large Eddy Simulation (2D) of a Reacting Plane Mixing Layer Using Filtered Density Function Closure. Flow, Turb. Comb. 2000; 64:279-300.

[23] Wang JK, Milane RE. Large eddy simulation (2D) of spatially developing mixing layer using vortex-in-cell for flow field and filtered probability density function for scalar field. Int. J. Num. Meth. Fluids 2006; 50:27-61.

[24] Ho C-M, Huang L-S. Subharmonics and vortex merging in mixing layers. $J$. Fluid Mech. 1982; 119:443-473.

[25] McMullan WA, Gao S, Coats CM. A comparative study of inflow conditions for two- and three-dimensional spatially developing mixing layers using Large Eddy Simulation. Int. J. Num. Meth. Fluids 2006; 55:589-610.

[26] Wang, Y, Tanahashi, M, Miyauchi, T. Coherent fine scale eddies in turbulence transition of spatially-developing mixing layer Int. J. Heat Fluid Flow 2007; 28:1280-1290.

[27] Vreman B, Geurts B, Kuerten H. Large-eddy simulation of the turbulent mixing layer. J. Fluid Mech. 1997; 339:357-390.

[28] de Bruin ICC. Direct and Large Eddy Simulation of the spatial turbulent mixing layer. $\mathrm{PhD}$ thesis, University of Twente, 2001.

[29] Comte P, Silvestrini JH, Bégou P. Streamwise vortices in Large Eddy Simulations of mixing layers. Eur. J. Mech. B/Fluids 1998; 4:615-637.

[30] Lo S, Voke PR, Rockliff NJ. Three dimensional vortices of a spatially developing plane jet. Int. J. Fluid Dyn. 2000; 4:319-336. 
[31] Gough T, Gao S, Hancock PE, Voke PR. Experiment and simulation of a tripped boundary layer on a flat plate: A comparative study. University of Surrey Internal Report, 1996

[32] Roache PJ. Computational Fluid Dynamics. Hermosa Publishers, 1972.

[33] Germano M, Piomelli U, Moin P, Cabot W. A dynamic subgrid-scale eddy viscosity model. Phys. Fluids A 1991; 3:1760-1765.

[34] Lilly DK. A proposed modification of the Germano subgrid-scale closure method. Phys. Fluids A 1992; 4:633-635.

[35] Smagorinsky J. General circulation experiments with the primitive equations. Mon. Weather Rev. 1963; 91:99-164.

[36] Gao S, Voke PR. Large Eddy Simulations of turbulent heat transport in enclosed impinging jets. Int. J. Heat Fluid Flow 1995; 16:349-356.

[37] Voke PR, Potamitis SG. Numerical simulations of a low-Reynolds-number turbulent wake behind a flat plate. Int. J. Num. Meth. Fluids 1994; 19:377-393.

[38] Soteriou, MC, Ghoniem, AF. On the Effects of Inlet Boundary Conditions on the Mixing and Burning in Reacting Shear Flows. Combustion and Flame 1998; 112:404-417

[39] Monkewitz, PA, Heurre, P. Influence of the velocity ratio on the spatial instability of mixing layers. Phys. Fluids 1982; 25:1137-1143

[40] Koochesfahani MM, Frieler CE. Instability of nonuniform density free shear layers with a wake profile. AIAA J. 1989; 27,:1735-1740

[41] D'Ovidio A. Coherent structures in turbulent mixing layers. $\mathrm{PhD}$ thesis, University of Leicester, 1998.

[42] Jimenez J. A spanwise structure in the plane mixing layer. J. Fuild Mech. 1983; 139:319-336.

[43] McMullan, WA. Numerical Experiments on Spatially Developing Mixing Layers Using Large Eddy Simulation. PhD thesis, University of Leicester, 2005.

[44] Karasso, PS, Mungal, MG Scalar mixing and reaction in plane liquid shear layers J. Fluid Mech. 1996; 323:23-63.

[45] D'Ovidio A, Coats CM. Coherent-structure evolution in turbulent mixing layers. Part 1: Experimental evidence. Under Review

[46] McMullan WA, Gao S, Coats CM. Coherent-structure evolution in turbulent mixing layers. Part 2: Large Eddy simulation. In preparation

[47] Lessen M, Ko S-H. Viscous Instability of an Incompressible Fluid Half-Jet Flow. Phys Fluids 1966; 9:1179-1183.

[48] Piomelli U. Large-eddy simulation: achievements and challenges. Prg. Aero. Sci. 1999, 35:335-362 


\begin{tabular}{ccc}
\hline Case & $\mathrm{L}_{x} \times \mathrm{L}_{y} \times \mathrm{L}_{z}$ & $\mathrm{~N}_{x} \times \mathrm{N}_{y} \times \mathrm{N}_{z}$ \\
\hline Wang et al. $[26]$ & $30 \Lambda \times 6 \Lambda \times 8 / 3 \Lambda$ & $2160 \times 433 \times 192$ \\
HR & $25 \Lambda \times 13 / 2 \Lambda \times 5 \Lambda$ & $1024 \times 200 \times 64$ \\
IM & $25 \Lambda \times 13 / 2 \Lambda \times 5 \Lambda$ & $256 \times 96 \times 64$ \\
\hline
\end{tabular}

Table 1

Grid parameters. 


\begin{tabular}{cccccc}
\hline Expt. Number & $U_{1}\left(\mathrm{~ms}^{-1}\right)$ & $U_{2}\left(\mathrm{~ms}^{-1}\right)$ & $R$ & $\theta_{1}\left(\times 10^{-4} \mathrm{~m}\right)$ & $x_{t}\left(x_{i}^{*}\right)$ \\
\hline F9 & 31.3 & 13.0 & 0.42 & 1.2 & 8.2 \\
F58 & 19.9 & 6.8 & 0.49 & 1.9 & 8.09 \\
HW9 & 32.12 & 9.59 & 0.54 & 1.2 & - \\
\hline
\end{tabular}

Table 2

Flow parameters of the simulated mixing layers. 


\begin{tabular}{ccc}
\cline { 2 - 3 } Simulation & $x_{t}\left(x_{i}^{*}\right)$ & $\operatorname{Re}\left(x_{t}\right)$ \\
\hline F9HTHR & 9.6 & 10046 \\
F9BLHR & 7.84 & 12387 \\
F58HTHR & 9.3 & 11285 \\
F58BLHR & 7.88 & 13534 \\
HW9HTHR & 9.4 & 14412 \\
HW9BLHR & 8.01 & 15666 \\
\hline
\end{tabular}

Table 3

Mean transition location, $x_{t}$, and local Reynolds number at the transition location for each HR grid simulation. 


\begin{tabular}{ccc}
\hline Simulation & $x_{t}\left(x_{i}^{*}\right)$ & $\operatorname{Re}\left(x_{t}\right)$ \\
\hline F9BLIM & 7.92 & 13779 \\
F58BLIM & 8.02 & 13920 \\
HW9BLIM & 8.2 & 17129 \\
\hline
\end{tabular}

Table 4

Mean transition location, $x_{t}$, and local Reynolds number at the transition location for BLIM simulations. 
(a)

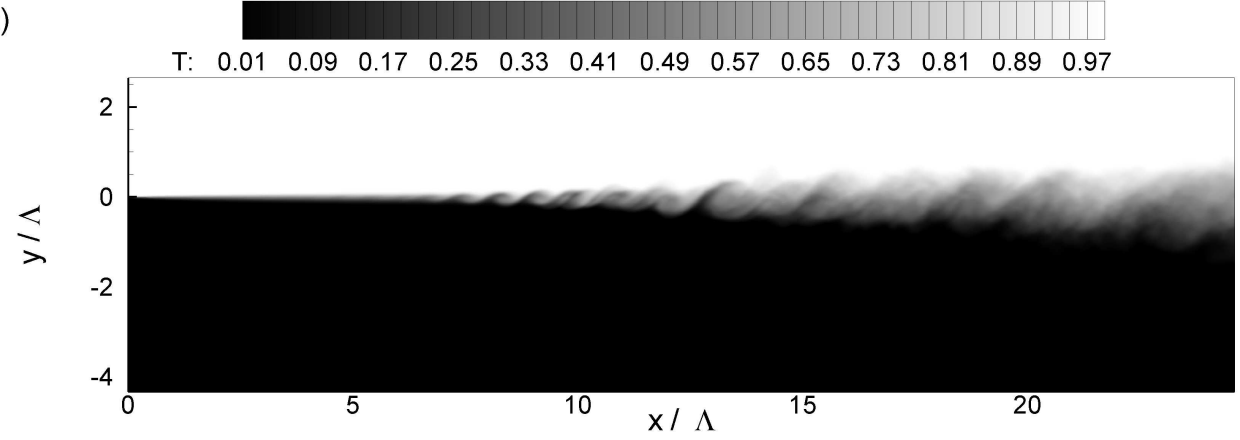

(b)

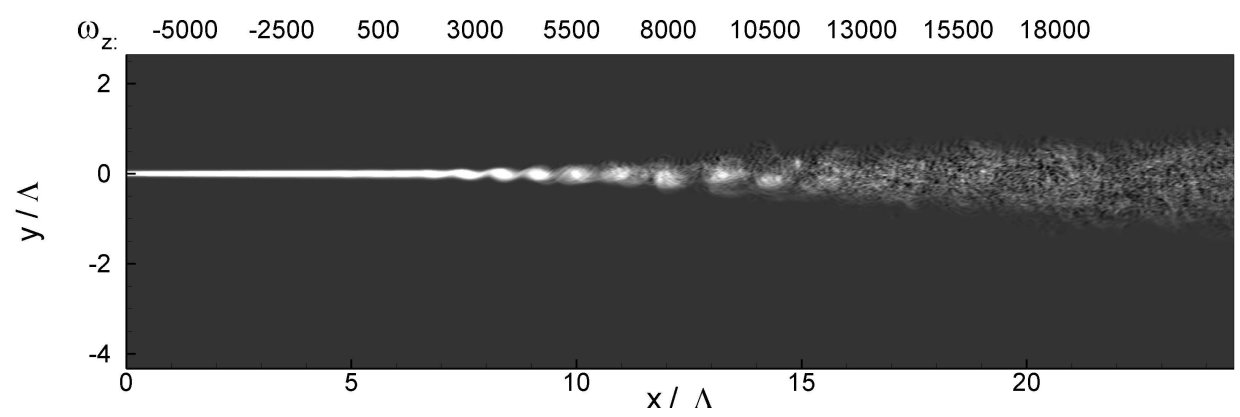

Fig. 1. Spanwise-averaged (a) passive scalar and (b) spanwise vorticity at a given time instant in case WHTHR. 


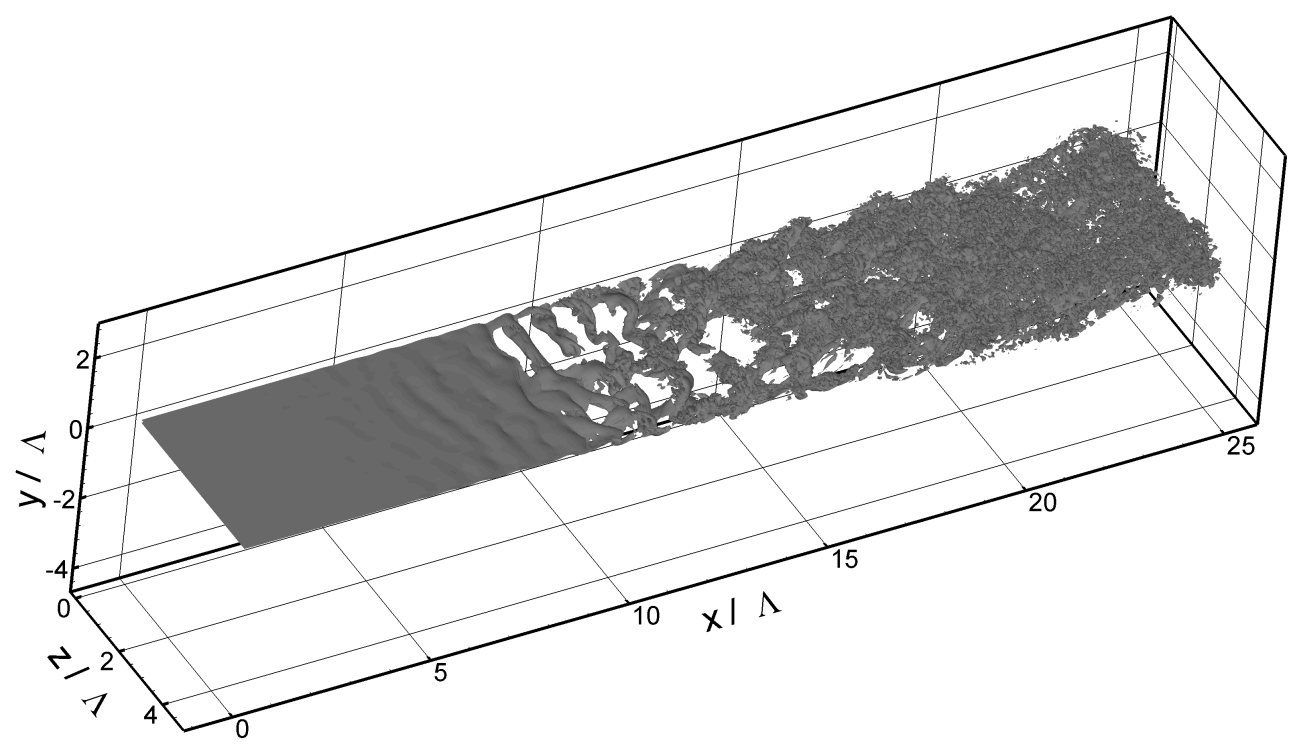

Fig. 2. Iso-surface of total vorticity in the flow, taken from case WHTHR. 


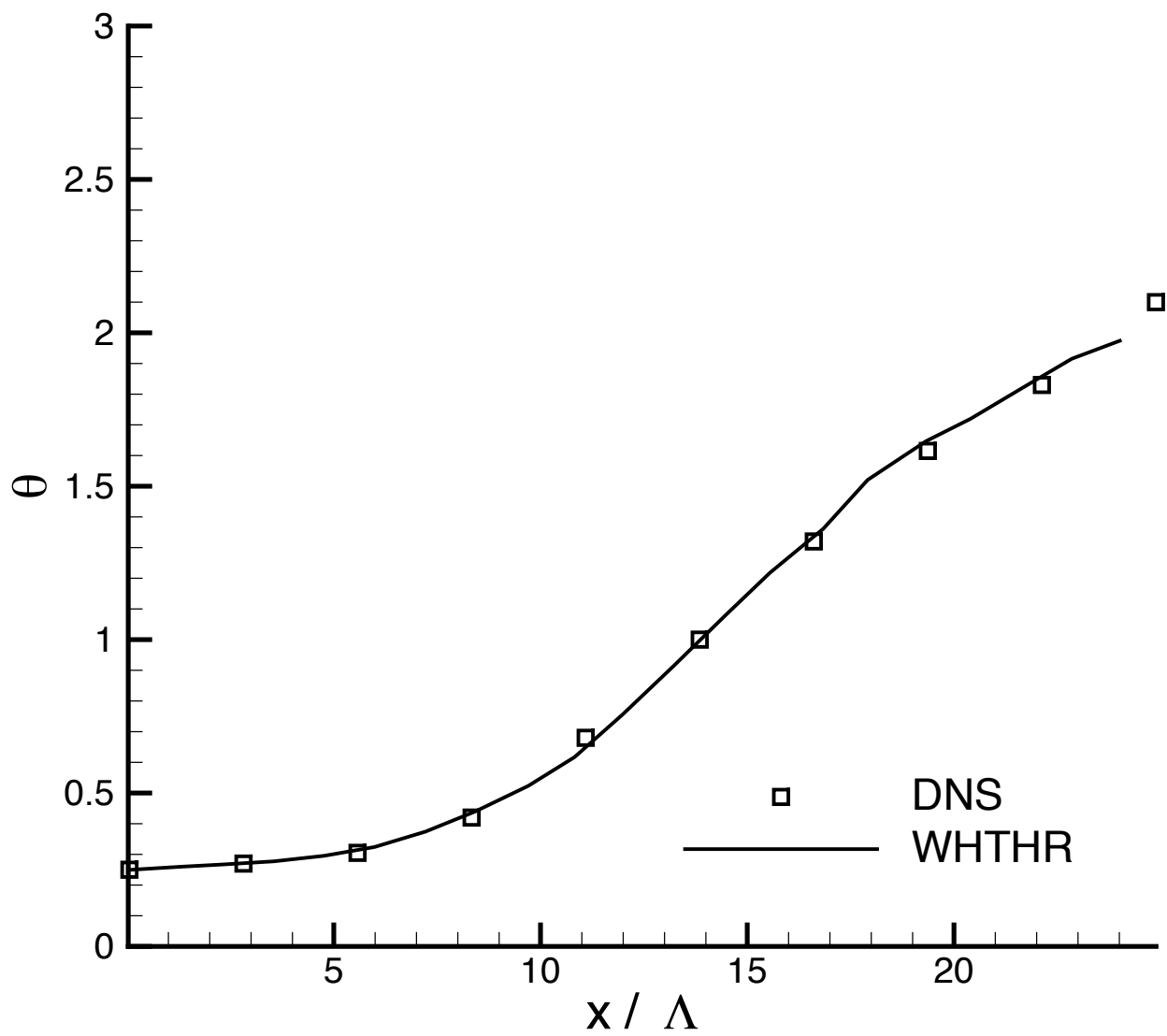

Fig. 3. Momentum thickness variation of case WHTHR. 


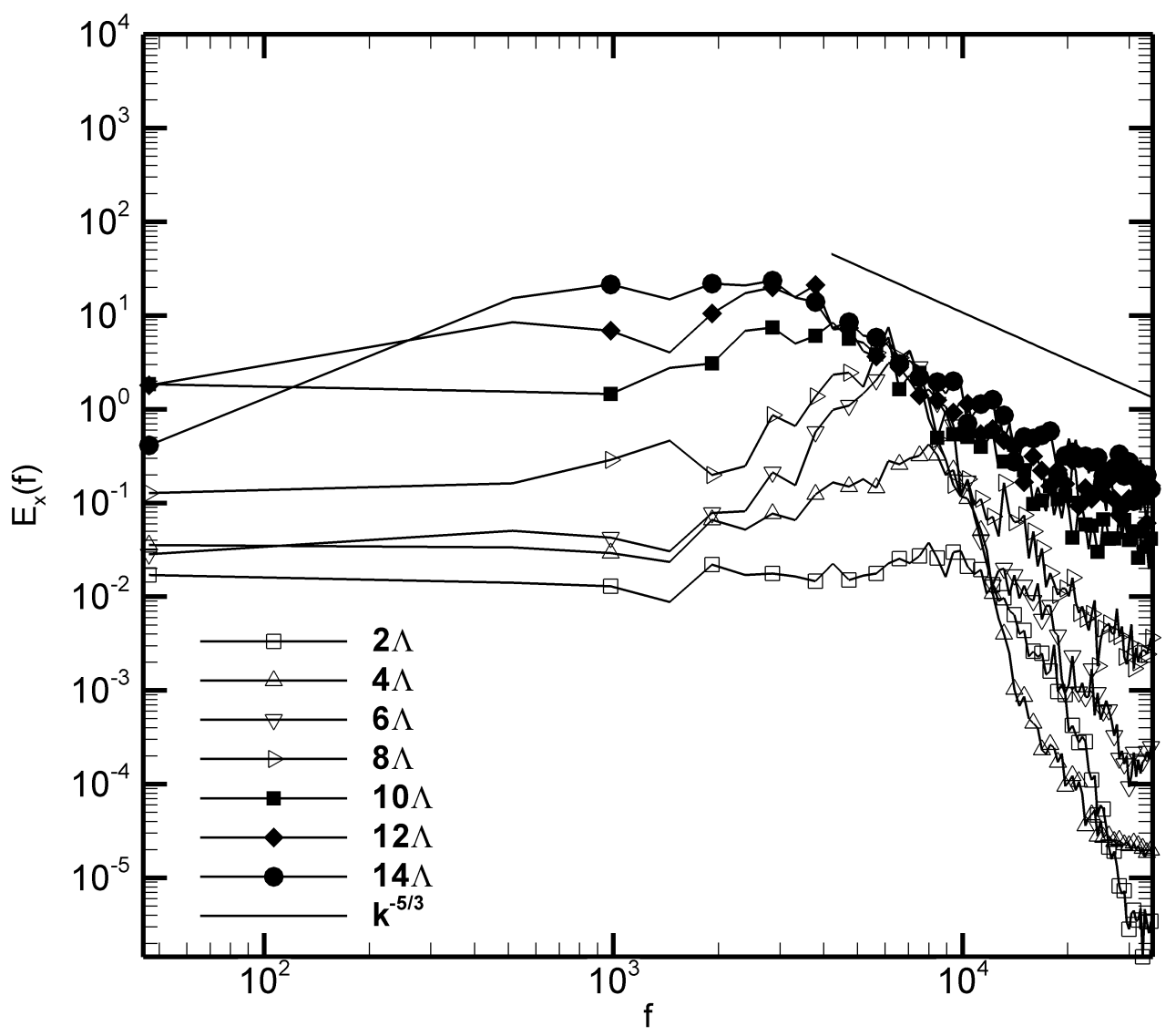

Fig. 4. Frequency spectra from case WHTHR recorded at several streamwise locations along the centreline of the flow. 


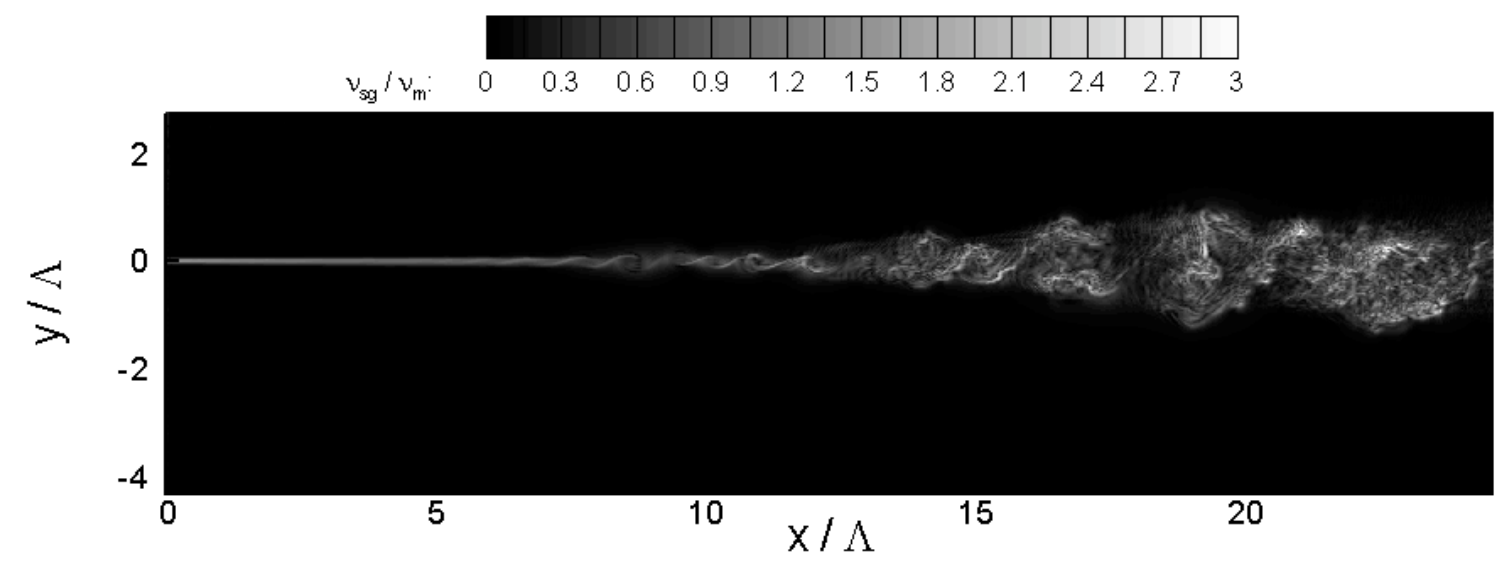

Fig. 5. Contours of the ratio of $\nu_{s g} / \nu_{m}$ at $z=0.015 \mathrm{~m}$ for case WHTHR. 


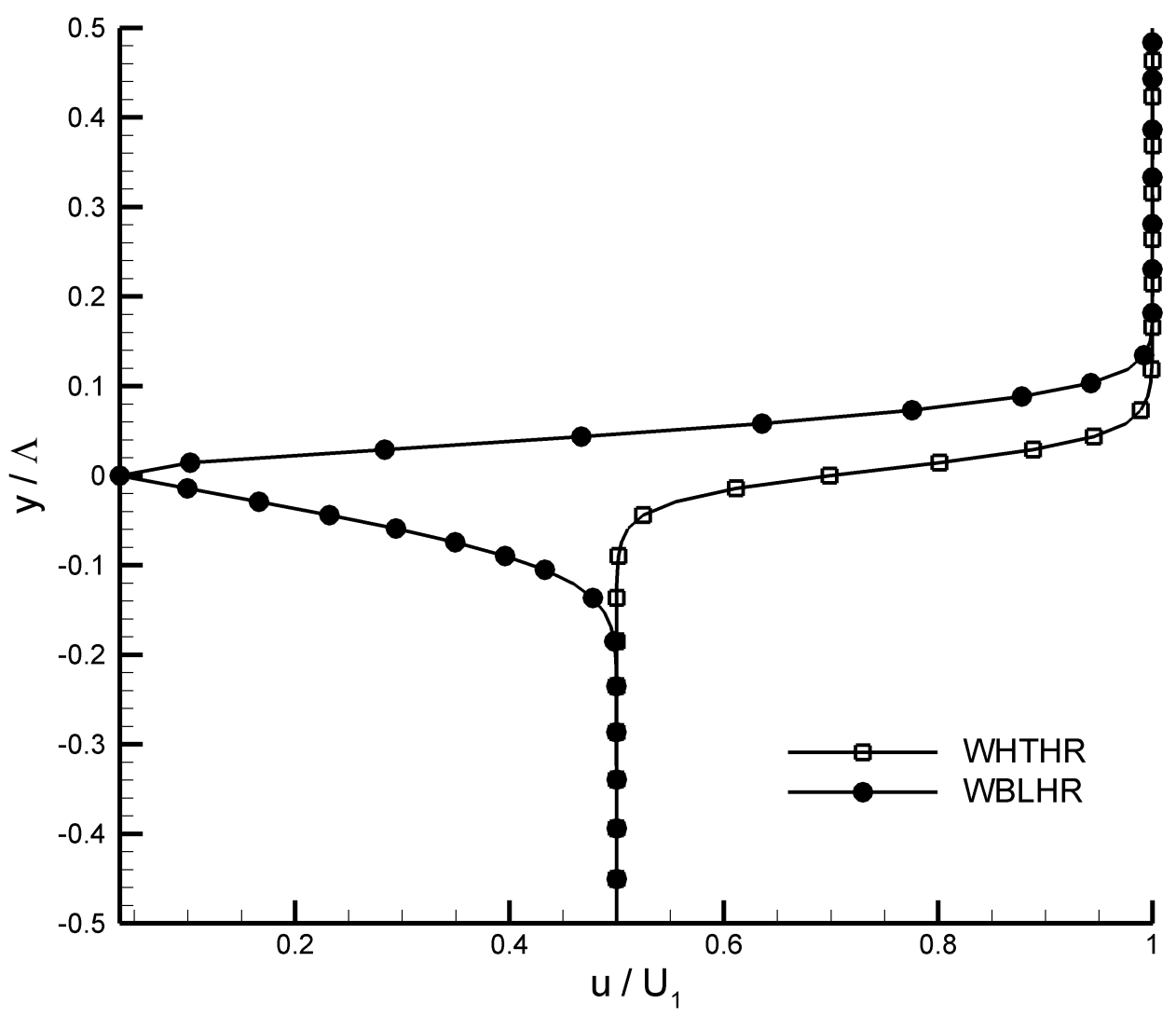

Fig. 6. Inflow profiles used in simulations WHTHR and WBLHR. 
(a)

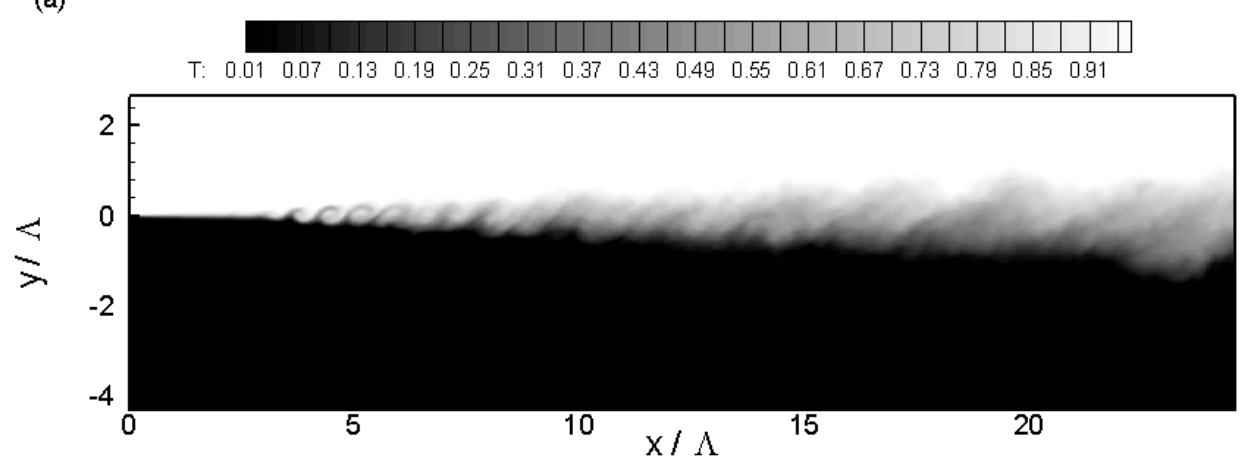

(b)

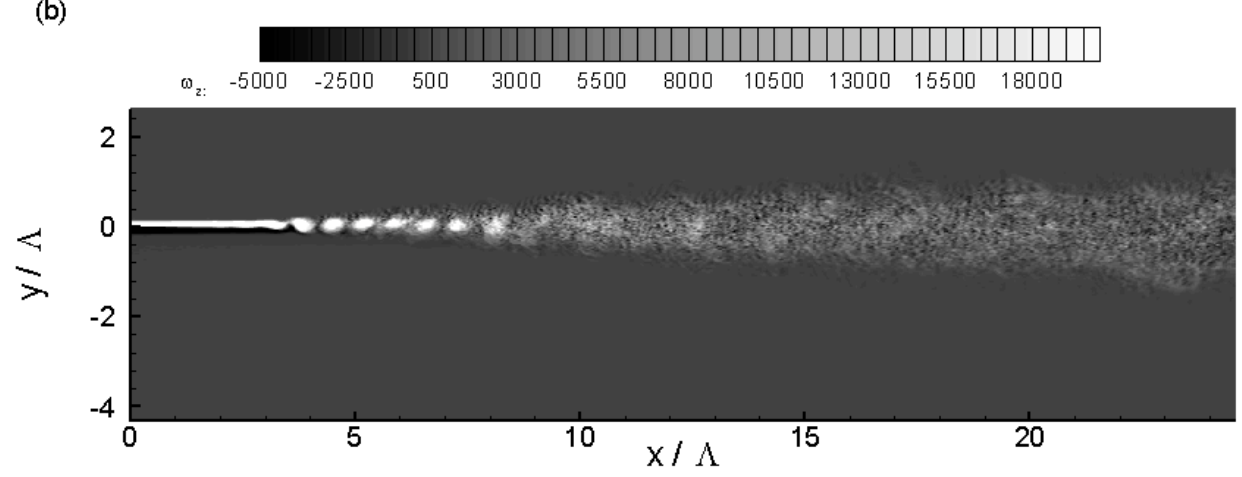

Fig. 7. Spanwise-averaged (a) passive scalar and (b) spanwise vorticity at a given time instant in case WBLHR. 


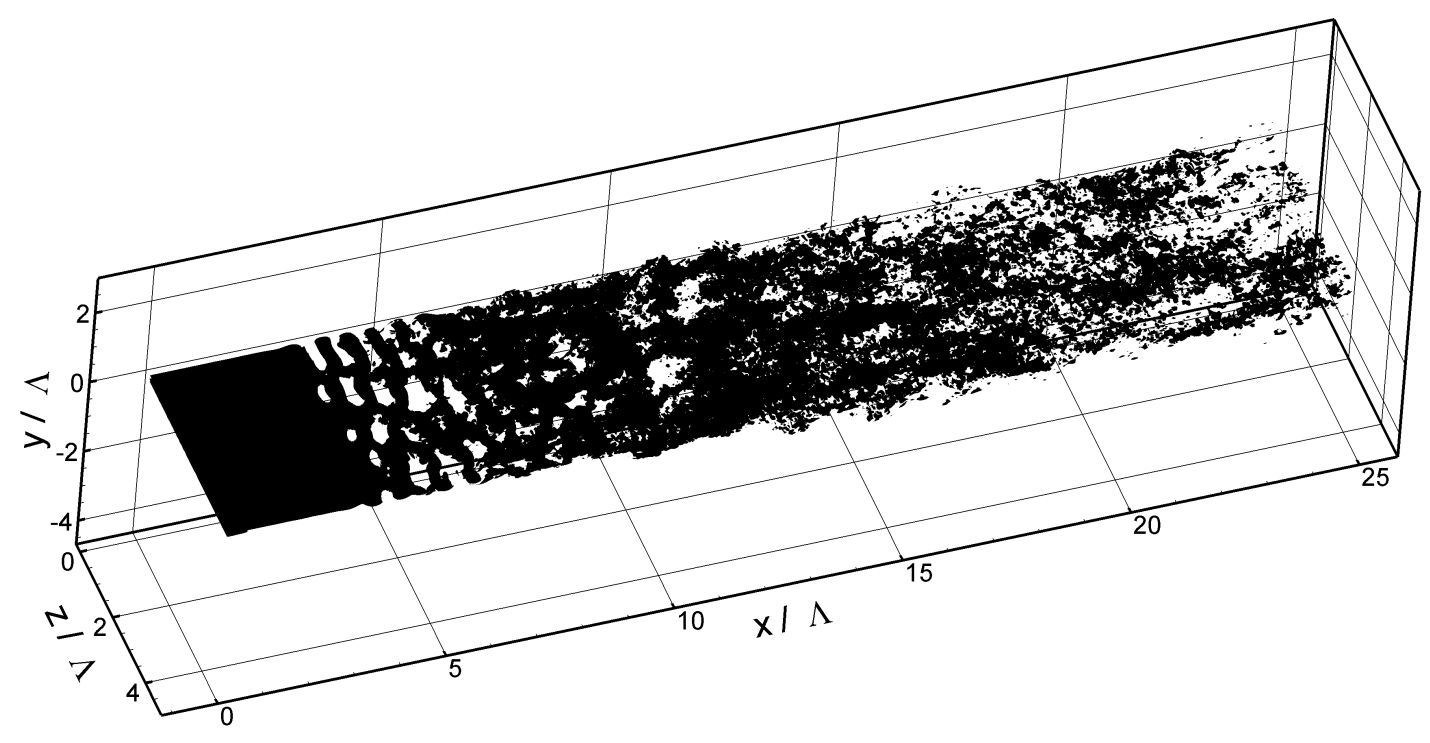

Fig. 8. Iso-surface of total vorticity in the flow, taken from case WBLHR. 


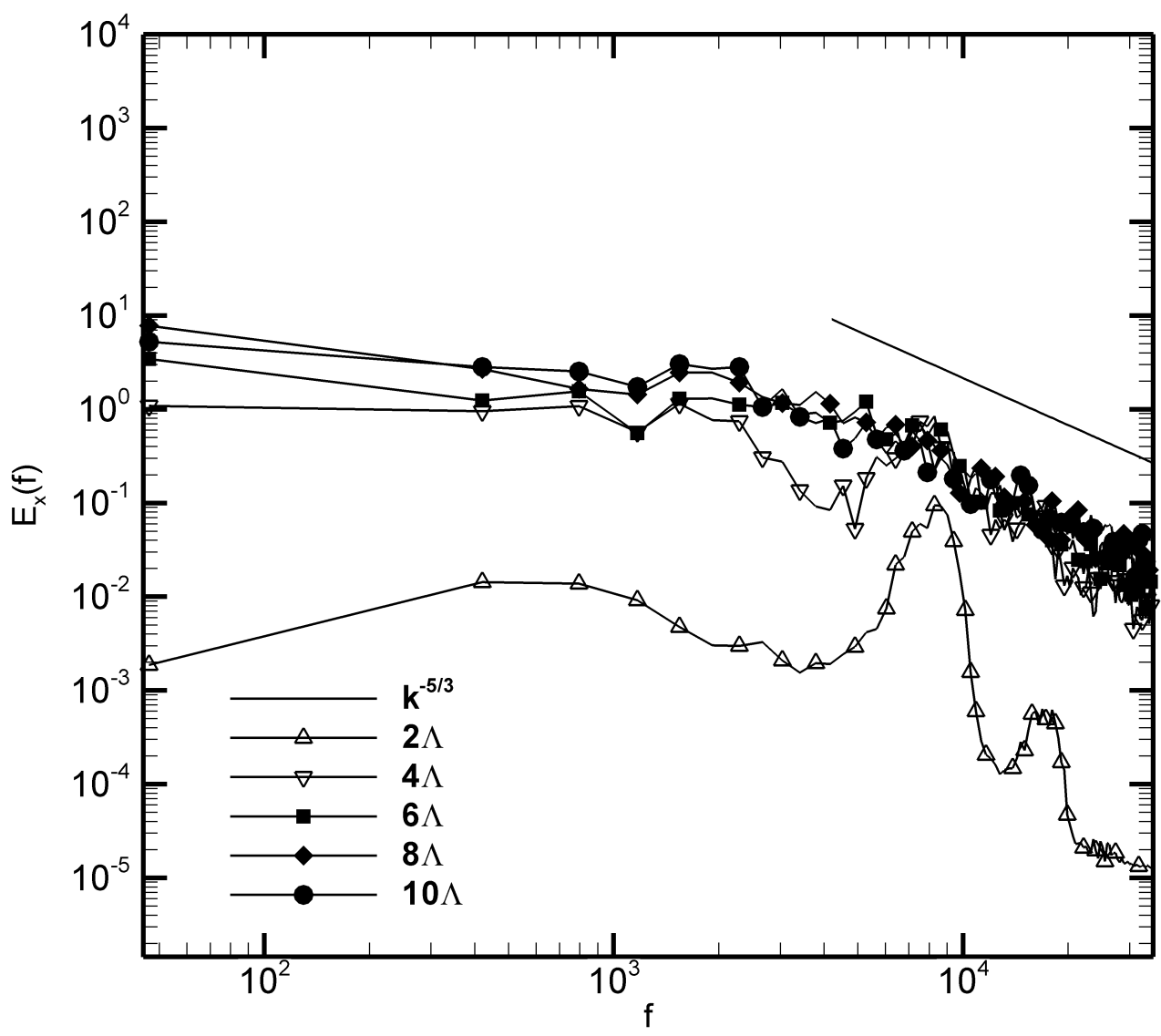

Fig. 9. Frequency spectra from case WBLHR recorded at several streamwise locations along the centreline of the flow. 
(a)

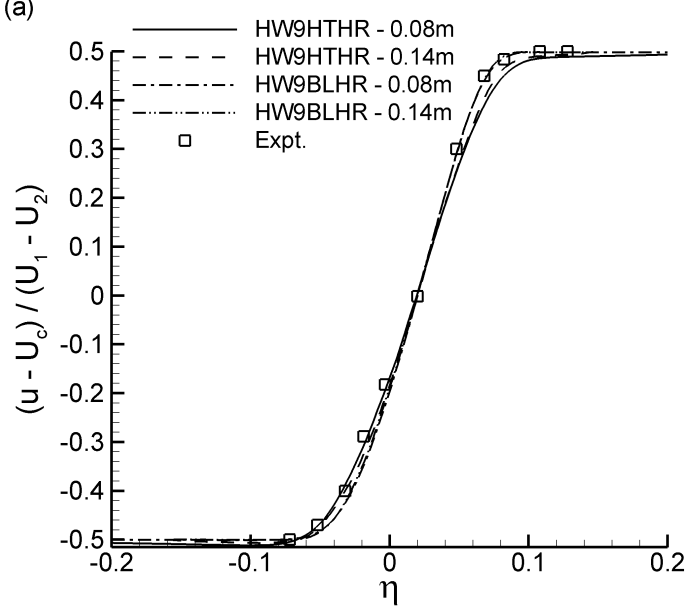

(c)

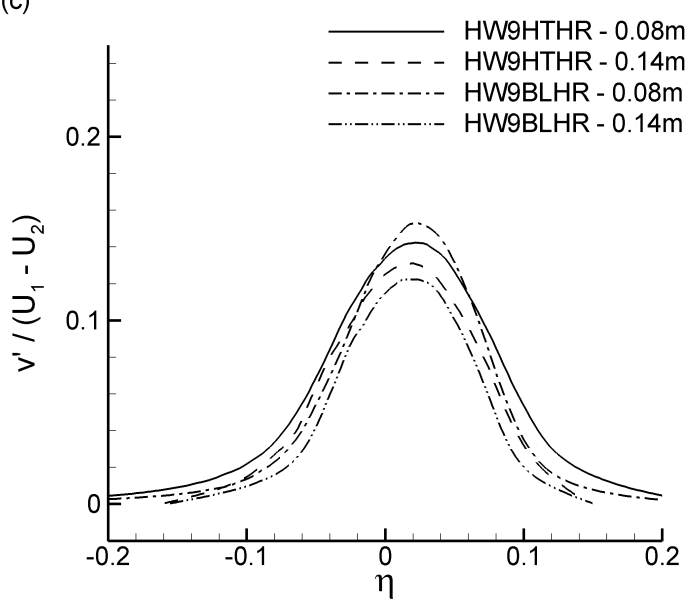

(b)

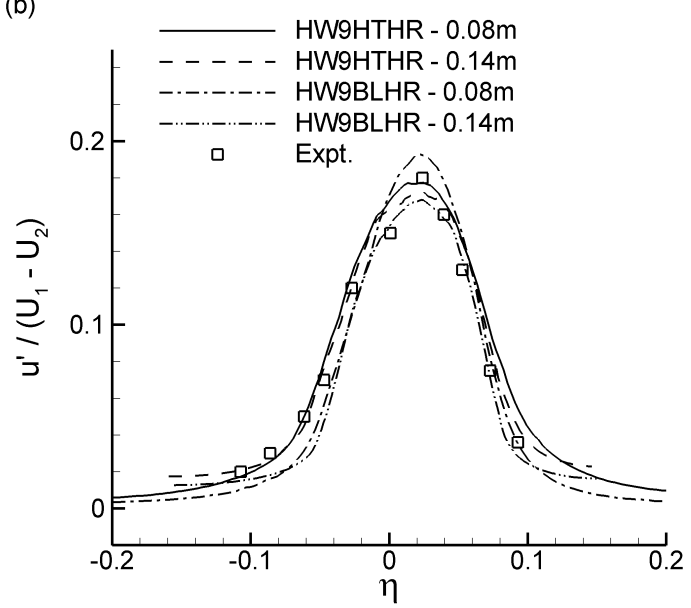

(d)

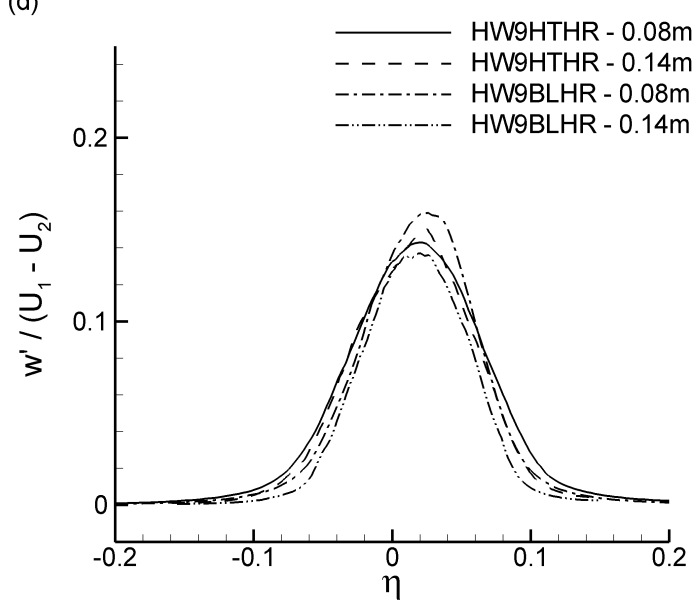

Fig. 10. Flow statistics for cases HW9HTHR and HW9BLHR. (a) mean streamwise velocity, (b) r.m.s. streamwise velocity fluctuation, (c) r.m.s. cross-stream velocity fluctuation, (d) r.m.s. spanwise velocity fluctuation. 


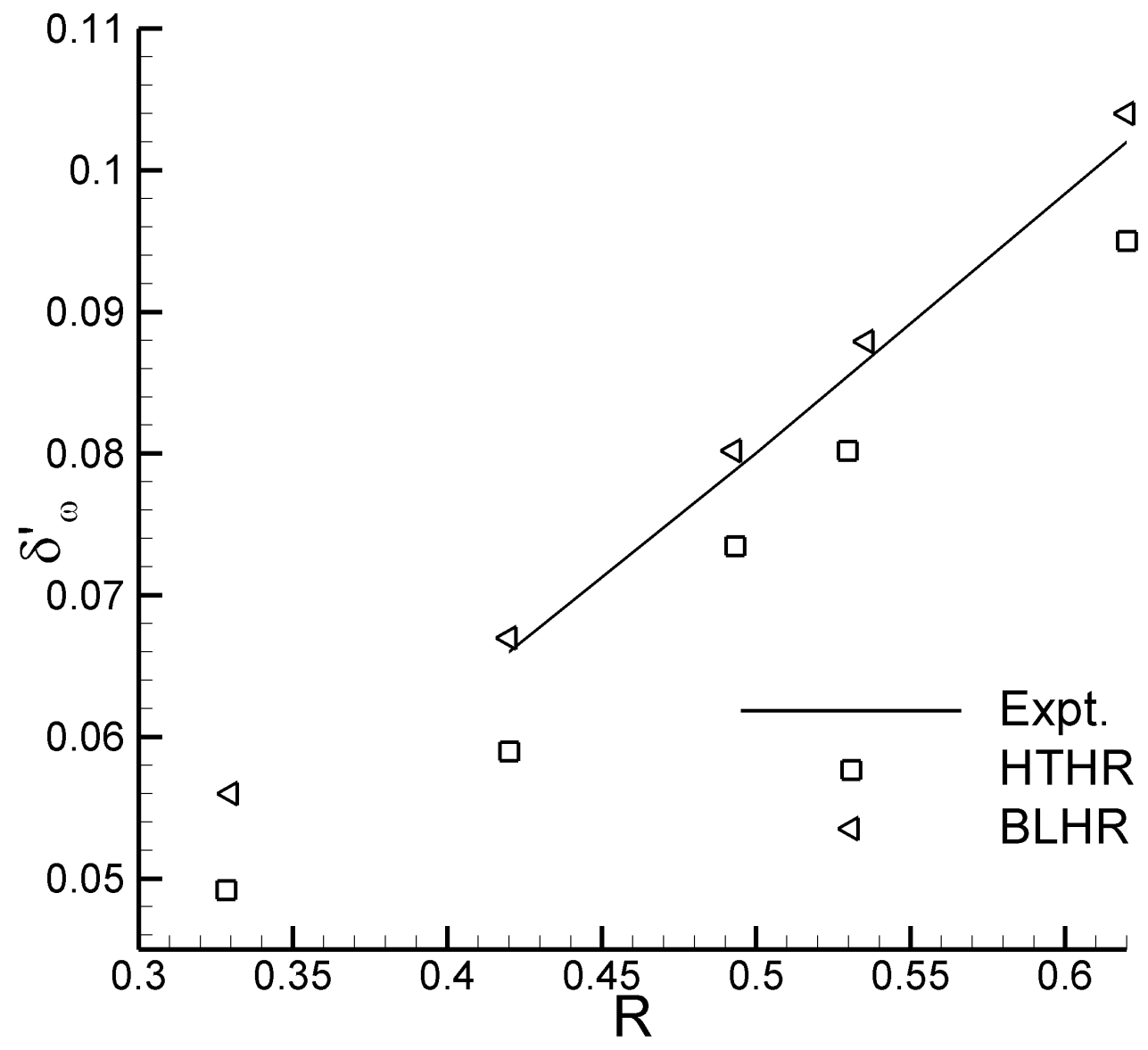

Fig. 11. Vorticity rate of the flows simulated on the High Resolution grid. 
(a)

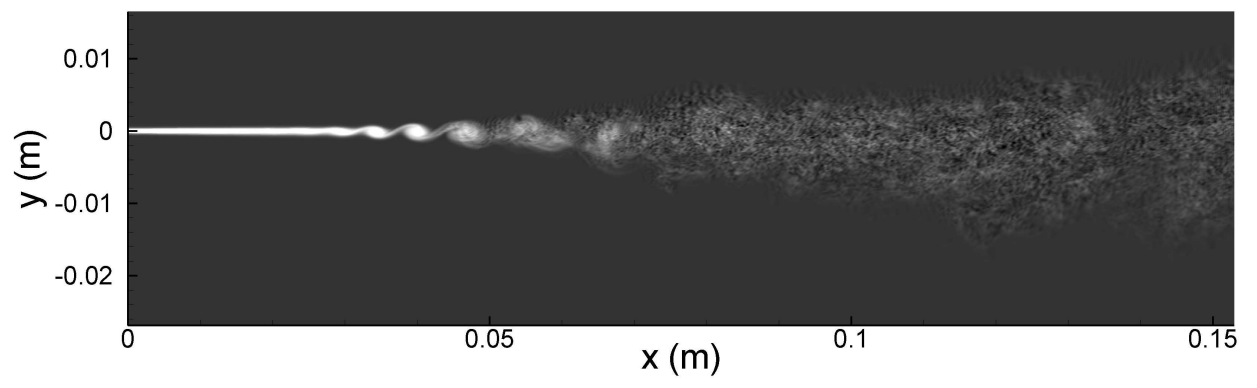

(b)

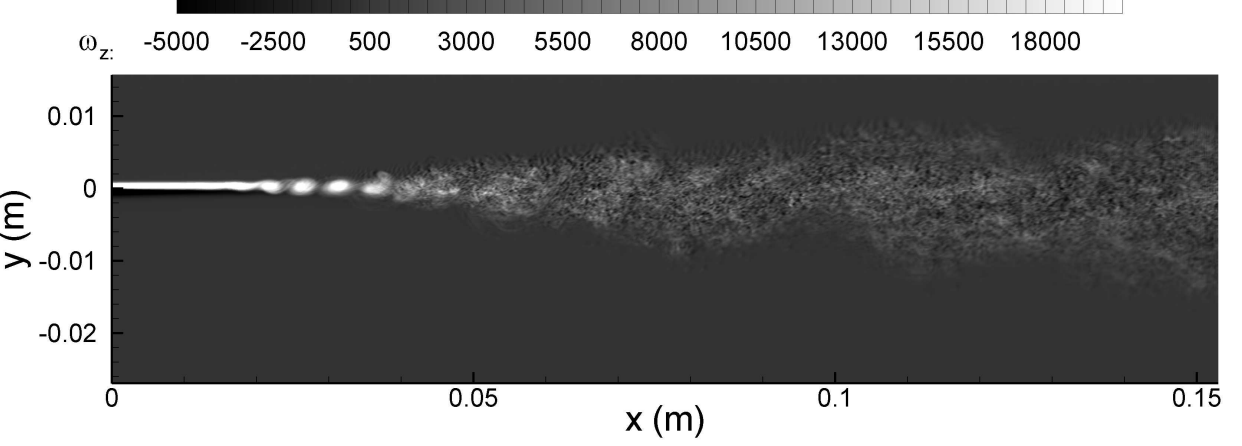

Fig. 12. Spanwise-averaged spanwise vorticity plots from case (a) HW9HTHR, and (b) HW9BLHR. 


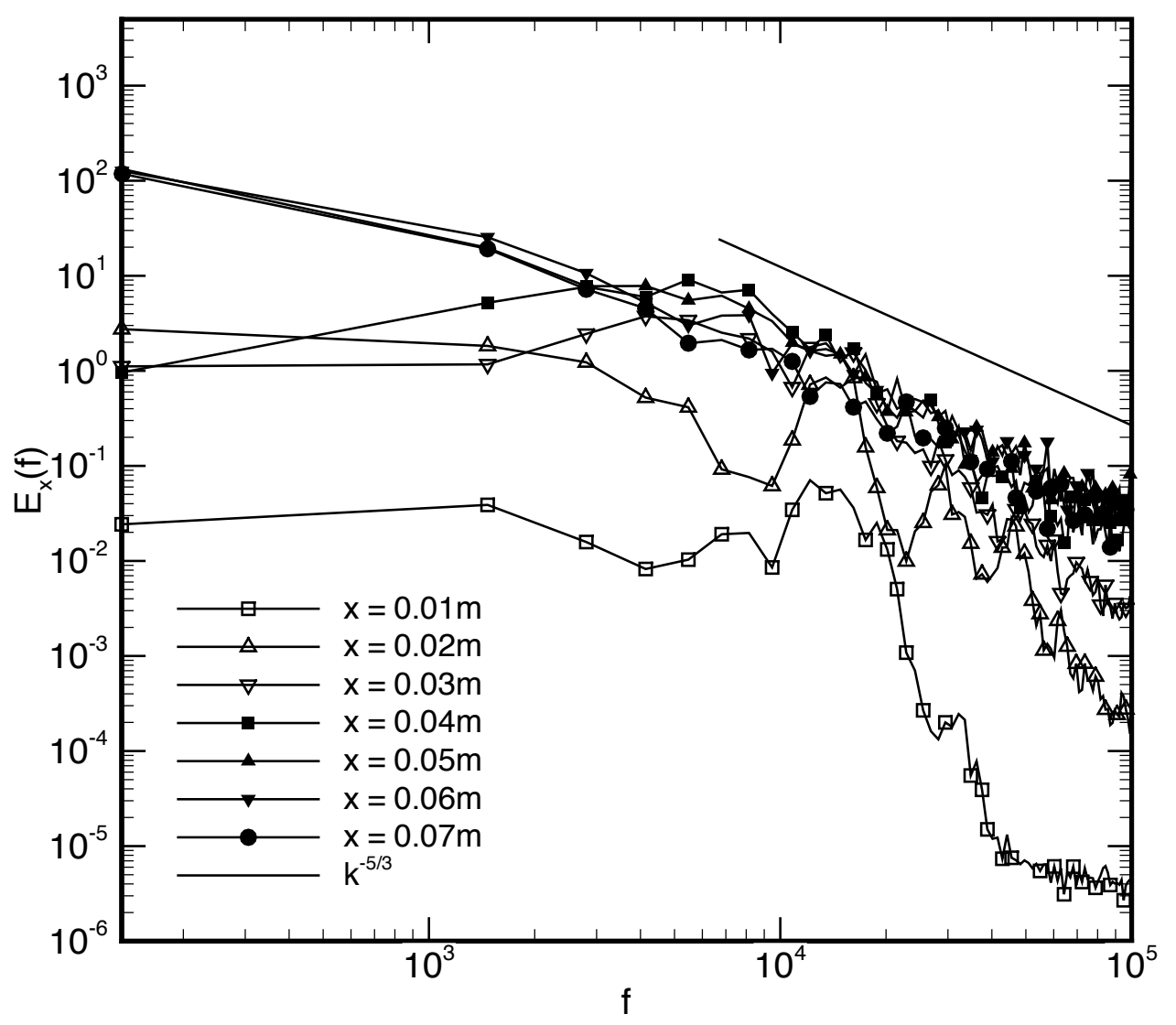

Fig. 13. Frequency spectra from case HW9BLHR recorded at several streamwise locations along the centreline of the flow. 


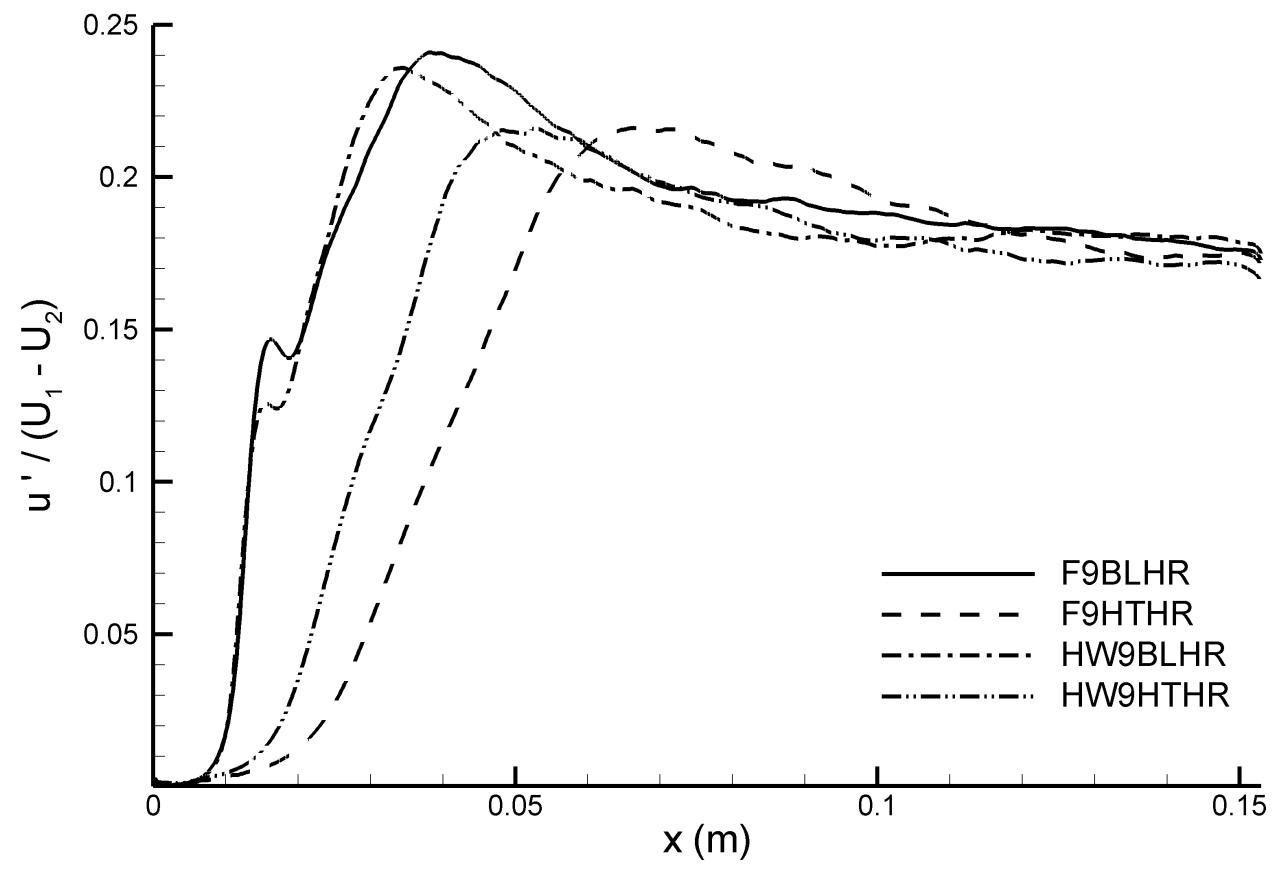

Fig. 14. Evolution of streamwise velocity fluctuation $u^{\prime}$ with streamwise distance on the HR grid. 
(a)

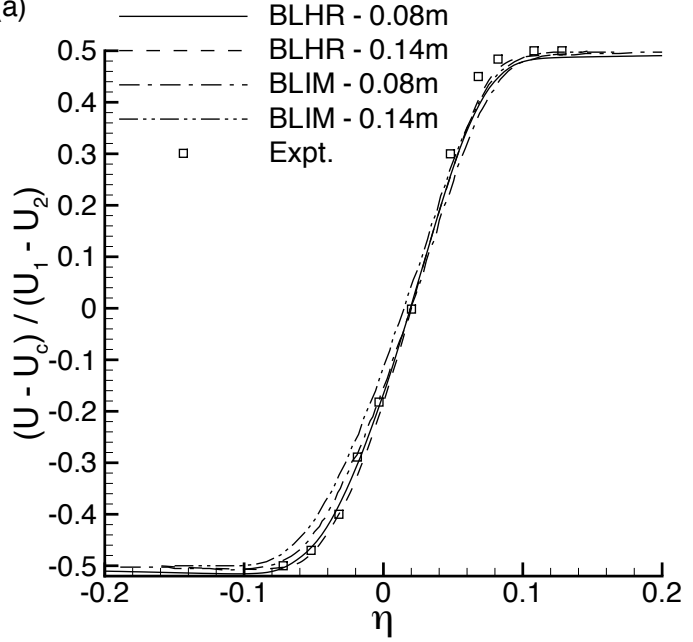

(c)

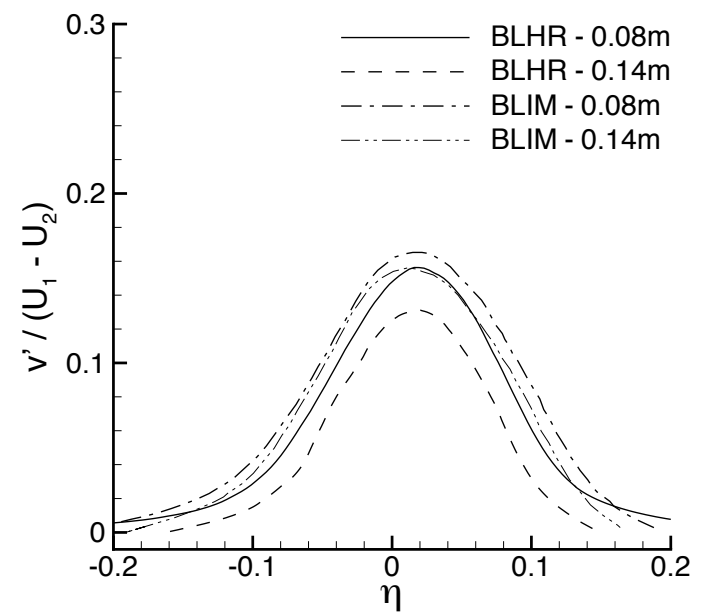

(b)

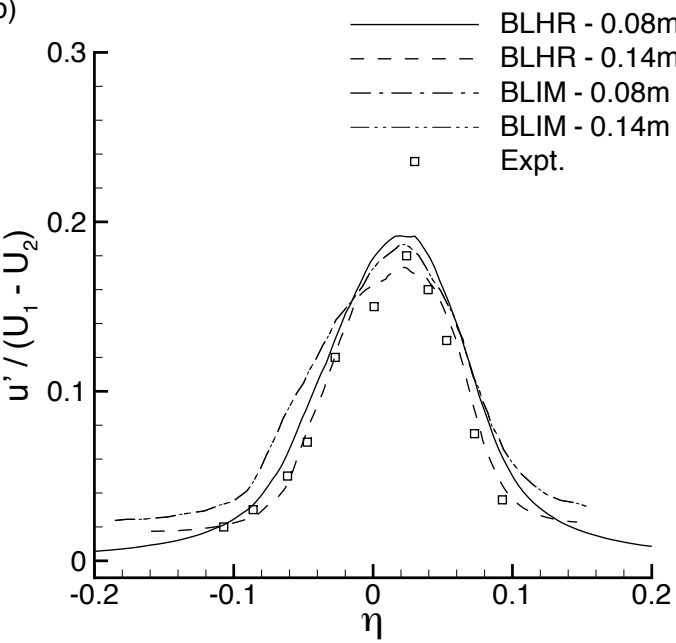

(d)

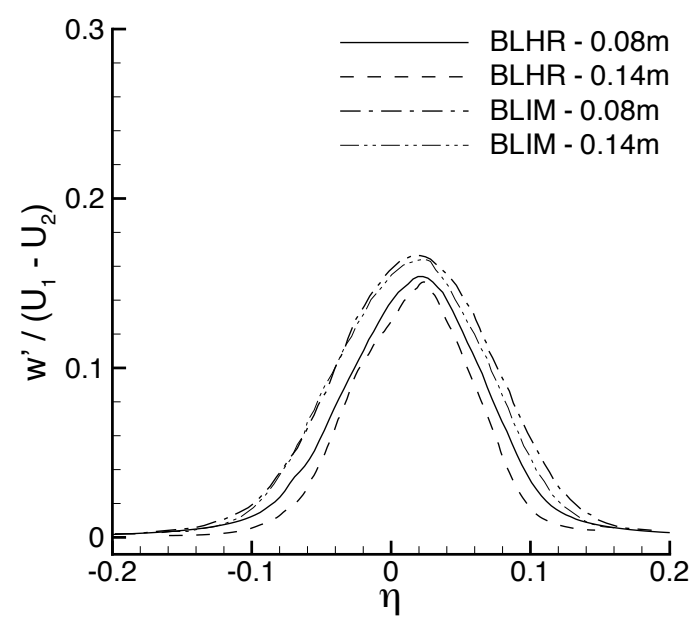

Fig. 15. Flow statistics for case HW9 on the IM grid. (a) mean streamwise velocity, (b) r.m.s. streamwise velocity fluctuation, (c) r.m.s. cross-stream velocity fluctuation, (d) r.m.s. spanwise velocity fluctuation. Results from HR grid shown for comparison. 

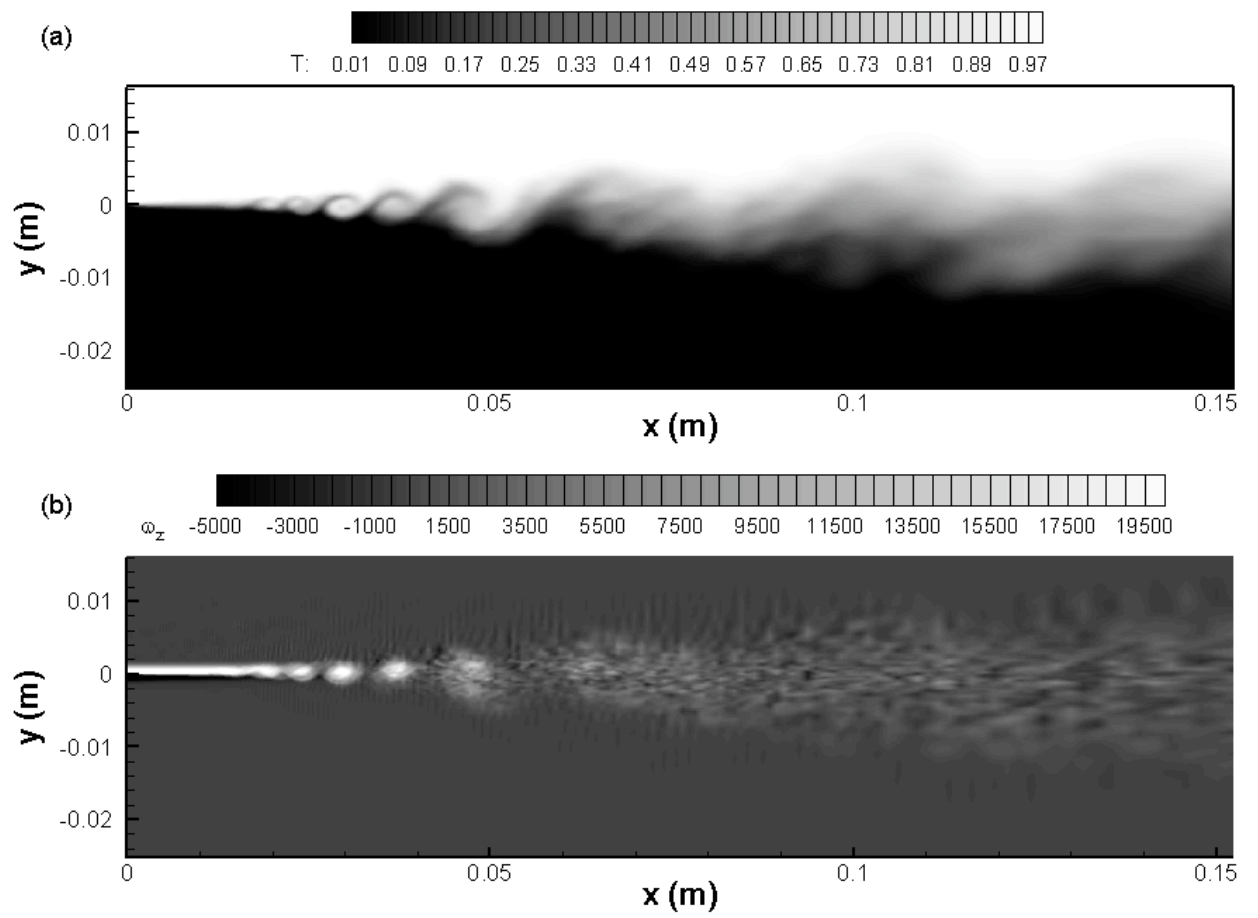

Fig. 16. Spanwise-averaged (a) passive scalar and (b) spanwise vorticity at a given time instant in case HW9BLIM. 


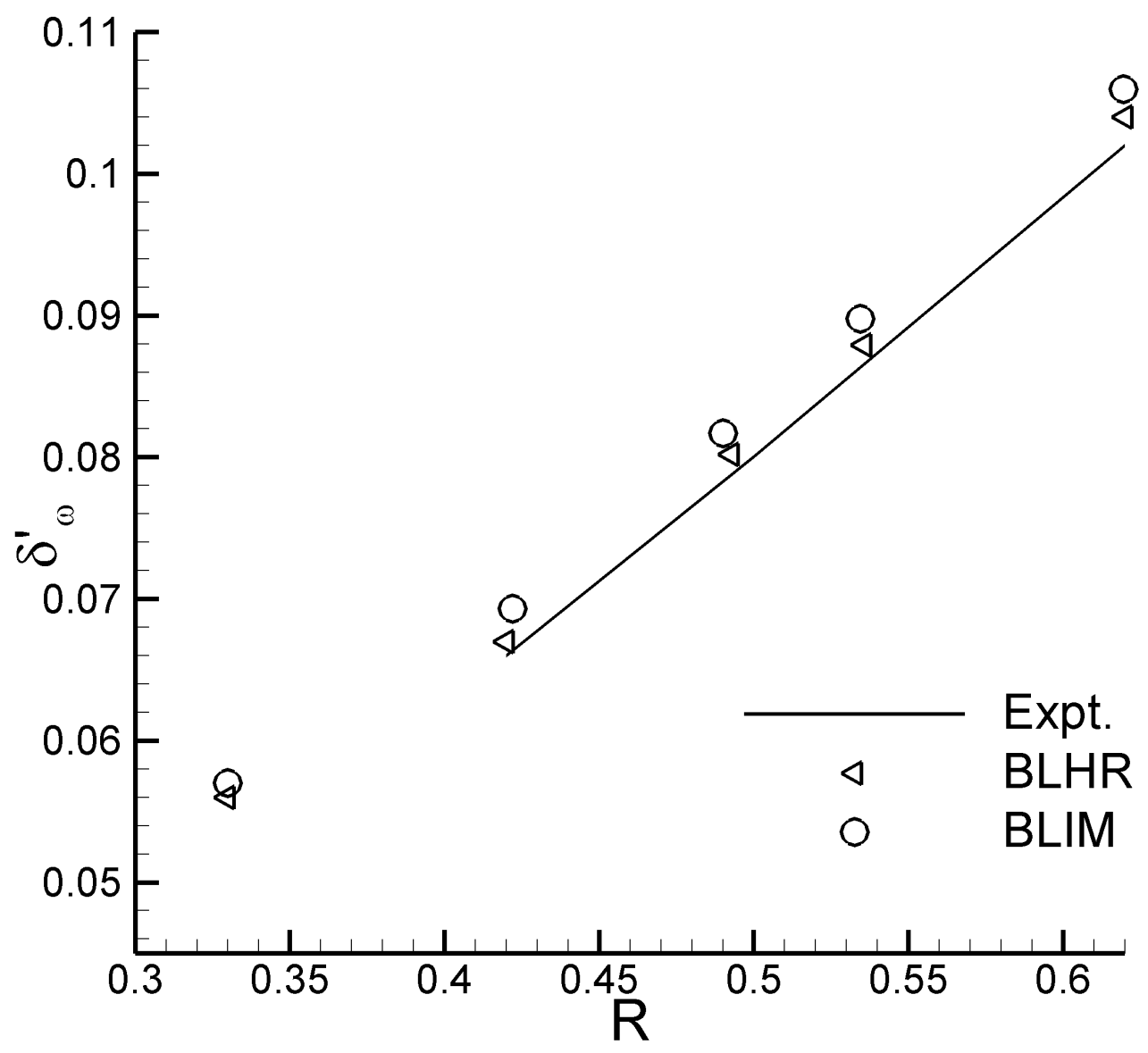

Fig. 17. Vorticity rate of the flow simulated on the IM grid. 


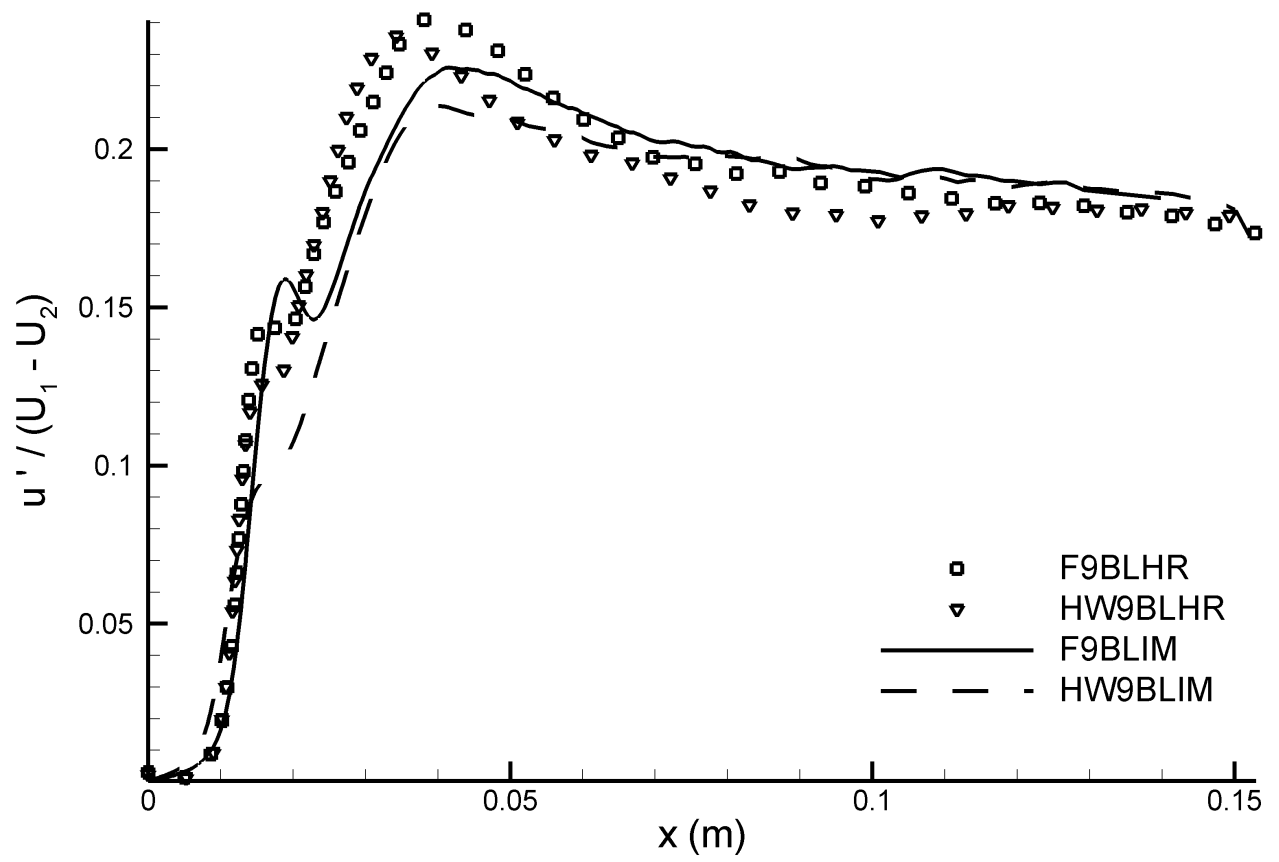

Fig. 18. Evolution of streamwise velocity fluctuation $u^{\prime}$ with streamwise distance on the IM grid. 


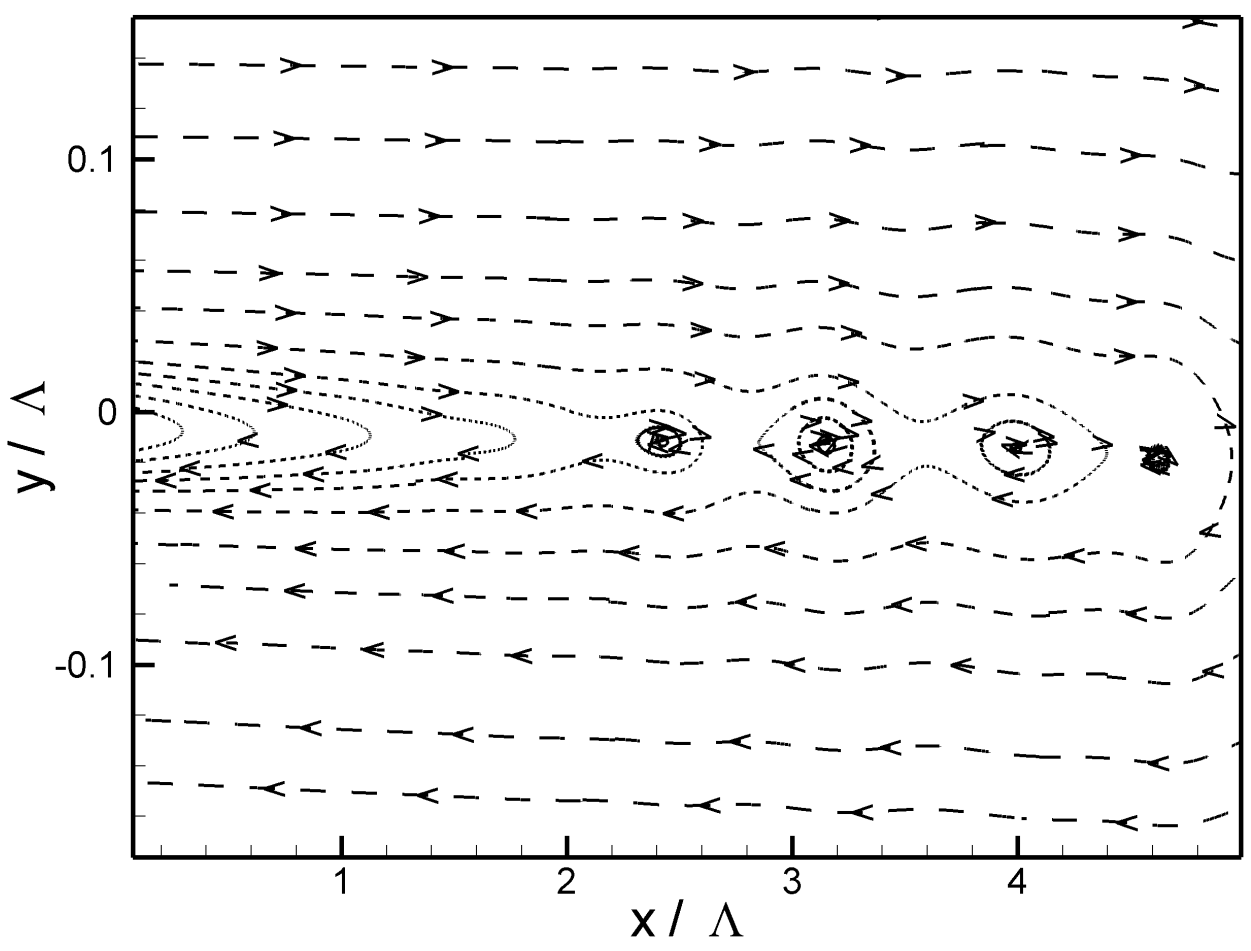

Fig. 19. Streamline plot showing roll-up of the flow in case WHTHR. 


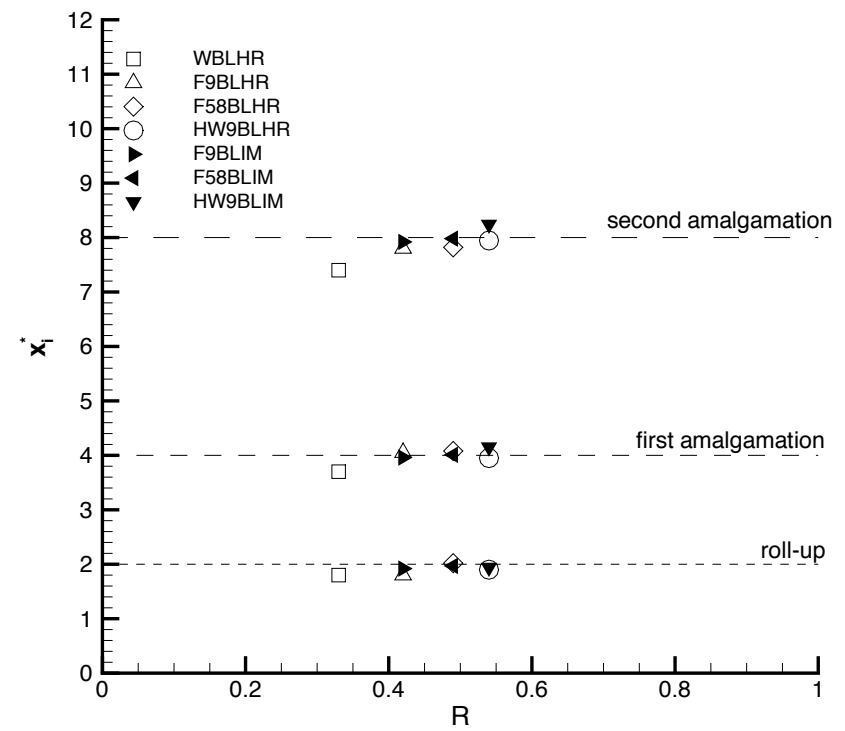

(a) Boundary layer inflow

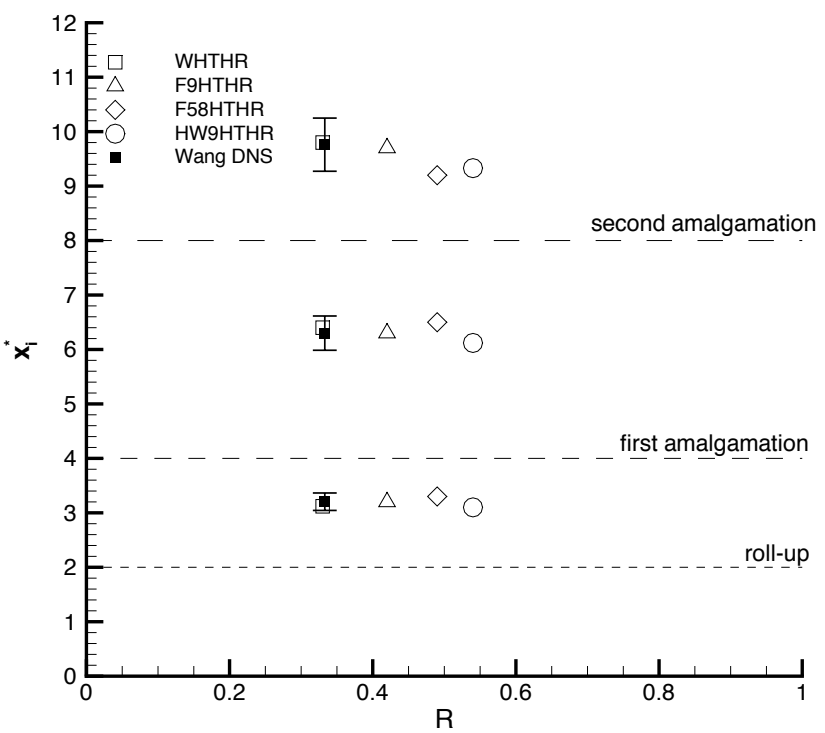

(b) Hyperbolic tangent inflow

Fig. 20. Mean locations of vortex events in the simulations 